\title{
A closer look at chaotic advection in the stratosphere: part I: geometric structure
}

Article

Published Version

Ngan, K. and Shepherd, T. G. (1999) A closer look at chaotic advection in the stratosphere: part I: geometric structure. Journal of the Atmospheric Sciences, 56 (24). pp. 4134-4152. ISSN 1520-0469 doi: https://doi.org/10.1175/15200469(1999)056<4134:ACLACA>2.0.CO;2 Available at https://centaur.reading.ac.uk/32855/

It is advisable to refer to the publisher's version if you intend to cite from the work. See Guidance on citing.

Published version at: http://dx.doi.org/10.1175/1520-0469(1999)056<4134:ACLACA>2.0.CO;2

To link to this article DOI: http://dx.doi.org/10.1175/1520-

0469(1999)056<4134:ACLACA>2.0.CO;2

Publisher: American Meteorological Society

All outputs in CentAUR are protected by Intellectual Property Rights law, including copyright law. Copyright and IPR is retained by the creators or other copyright holders. Terms and conditions for use of this material are defined in the End User Agreement.

www.reading.ac.uk/centaur

\section{CentAUR}


Central Archive at the University of Reading

Reading's research outputs online 


\title{
A Closer Look at Chaotic Advection in the Stratosphere. Part I: Geometric Structure
}

\author{
KeIth NGAN* AND TheOdore G. SHePHerd \\ Department of Physics, University of Toronto, Toronto, Ontario, Canada
}

(Manuscript received 17 September 1997, in final form 22 February 1999)

\begin{abstract}
The relevance of chaotic advection to stratospheric mixing and transport is addressed in the context of (i) a numerical model of forced shallow-water flow on the sphere, and (ii) a middle-atmosphere general circulation model. It is argued that chaotic advection applies to both these models if there is suitable large-scale spatial structure in the velocity field and if the velocity field is temporally quasi-regular. This spatial structure is manifested in the form of "cat's eyes" in the surf zone, such as are commonly seen in numerical simulations of Rossby wave critical layers; by analogy with the heteroclinic structure of a temporally aperiodic chaotic system the cat's eyes may be thought of as an "organizing structure" for mixing and transport in the surf zone. When this organizing structure exists, Eulerian and Lagrangian autocorrelations of the velocity derivatives indicate that velocity derivatives decorrelate more rapidly along particle trajectories than at fixed spatial locations (i.e., the velocity field is temporally quasi-regular). This phenomenon is referred to as Lagrangian random strain.
\end{abstract}

\section{Introduction}

It has sometimes been said that chaotic advection occurs in the stratosphere, in the sense that there is exponential lengthening of material contours (Pierce and Fairlie 1993; Tuck et al. 1997). While this definition may be satisfactory in practice, from a theoretical perspective it would appear to be insufficiently restrictive: there is exponential lengthening of material contours in turbulent flows (e.g., Batchelor 1952), and even if one were to associate contour-lengthening rates with positive Liapunov exponents, positive Liapunov exponents by themselves are not proof of chaotic dynamics (e.g., Wiggins 1990), let alone chaotic advection. One therefore wonders if it is possible to speak precisely of chaotic advection in the stratosphere.

Yet chaotic advection is not generally regarded as a particularly ambiguous concept. It is now widely accepted in the fluid dynamics literature that chaotic advection describes a situation in which a regular but timedependent velocity field produces irregular particle trajectories (e.g., Ottino 1989). This has proven to be a satisfactory definition inasmuch as chaotic advection has been studied mostly in the context of kinematic

\footnotetext{
* Current affiliation: Department of the Geophysical Sciences, University of Chicago, Chicago, Illinois.
}

Corresponding author address: Dr. Keith Ngan, Department of Geophysical Sciences, University of Chicago, 5734 S. Ellis Avenue, Chicago, IL 60637.

E-mail:kngan@midway.uchicago.edu flows, for which the time dependence is externally prescribed. In these flows it is clear what the word "regular" is supposed to denote: the velocity field is spatially smooth and periodic or quasiperiodic in time. This is the case in industrial applications (e.g., Jana et al. 1994), where time dependence in the velocity field is generated through motion of the boundaries, transient effects being negligible due to the restriction to Stokes flow.

The difficulty with the application of chaotic advection to stratospheric mixing and transport is that one is dealing with a flow that is intrinsically dynamical. Consequently, the meaning of chaotic advection in this context is unclear: the velocity field may not be regular for the time dependence is not necessarily quasiperiodic. However, recent work by Malhotra and Wiggins (1998) shows that chaotic advection does generalize in a natural way to temporally aperiodic kinematic flows. They show that a mathematically precise meaning can be ascribed to chaotic advection in temporally aperiodic systems if there exists a well-defined heteroclinic structure associated with the (aperiodic) hyperbolic trajectories; in the same way as with quasiperiodic systems (e.g., Wiggins 1992), chaotic mixing and transport are then mediated by the lobes formed by the stable and unstable manifolds, the "template" for chaotic dynamics.

Of course application of this mathematically precise definition to an arbitrary dynamical flow is problematic. In the absence of a simple analytical expression for the velocity field, the manifolds must be computed numerically from, say, model output or meteorological analyses; given that such data are not "perfect" and generally of low resolution (so that the hyperbolic points 
may not be well defined), accurate numerical computation from finite time series is difficult. Nevertheless geometrical organization of the flow by some kind of large-scale structure should be robust. This then leads us to the following inference: a necessary condition for chaotic advection in dynamical flows is that there be suitable spatial structure in the velocity field, that is, if, by analogy with the heteroclinic structure of an aperiodic system, there exists an "organizing structure" (that includes stagnation points) for mixing and transport. This is in accord with chaotic advection being a manifestation of deterministic chaos - a phenomenon that emerges in time rather than space and time.

There is strong evidence for the existence of such spatial structure in the stratosphere. The seminal papers by Waugh and Plumb (1994) and Norton (1994) showed that small-scale tracer structure in the stratosphere is determined mostly by the large-scale flow. This behavior is consistent with the so-called Rossby wave criticallayer paradigm (e.g., Juckes and McIntyre 1987), which states that the forced Rossby wave critical layer provides a useful conceptual model for the stratospheric surf zone. ${ }^{1}$ It is supported by numerical simulations, as well as theoretical studies (Haynes and McIntyre 1987; Salby and Garcia 1987) and observational analyses (Bowman 1996).

While the existence of an organizing structure is a necessary condition for there to be chaotic advection, it is not, however, a sufficient one. Besides spatial organization of the velocity field in the sense described above, the velocity field must be "quasi-regular" as well: irregularity in the particle trajectories needs to arise in a Lagrangian rather than Eulerian sense, though periodicity or quasiperiodicity of the underlying flow is not required. As discussed for example by Swanson and Pierrehumbert (1997), chaotic advection may be thought of as a situation in which the Lagrangian correlation time is short compared to the Eulerian correlation time.

In this paper we examine the relevance of chaotic advection to stratospheric mixing and transport. Specifically, we analyze two models of the wintertime lower stratosphere: (i) a shallow-water model of the kind previously used to simulate a perturbed polar vortex (Juckes 1989; Salby et al. 1990a; Polvani et al. 1995) and (ii) a middle-atmosphere general circulation model (Beagley et al. 1997). The shallow-water model, though extremely idealized, is nonetheless analyzed in detail for it is generally believed that such a model does capture the essential aspects of polar vortex dynamics; moreover, its parameter dependence may be easily studied (sections 3-4). The middle-atmosphere general circulation model is analyzed (section 5) with a view to-

\footnotetext{
${ }^{1}$ It should be noted that this is not the only possible mechanism. Ishioka and Yoden (1995) have studied the mixing and transport due to a barotropically unstable polar vortex and emphasized the importance of the unperturbed separatrix structure.
}

ward confirming the robustness of the shallow-water results.

The analysis is primarily qualitative: a high-resolution visualization of the surf zone-and of the associated organizing structure-is obtained from offline trajectory calculations and, to a limited extent, from the potential vorticity fields. In cases where the forcing amplitude is not too strong (and external stochasticity weak), a "cat's eye" structure, of the kind seen in numerical simulations of the barotropic, $\beta$-plane, critical-layer equations (Haynes 1989), is clearly observed. This cat's eye structure organizes mixing and transport in the surf zone, the determination of small-scale tracer structure by the large-scale flow being a direct consequence. The quasiregularity of the velocity field is established from Eulerian and Lagrangian autocorrelations of the velocity derivatives (section 4). Though the correlation times cannot be determined precisely, it is clear that the Lagrangian correlation time is much shorter than the Eulerian one; we refer to this as Lagrangian random strain. We comment on the relationship between chaotic advection, random-strain theory, and stratospheric mixing and transport.

A quantitative analysis of mixing and transport is not undertaken in this paper. This is left to the companion paper, Ngan and Shepherd (1999, hereafter referred to as Part II), where various statistical diagnostics are computed and comparisons with kinematic models of chaotic advection made. In that paper some practical implications of chaotic advection for stratospheric mixing and transport are considered; in this paper we shall mostly be concerned with theoretical issues. Since chaotic advection offers, at present, one of the few systematic approaches to mixing and transport in complicated but not fully turbulent flows, ${ }^{2}$ a reexamination of its relevance to stratospheric mixing and transport may be of some value.

\section{Shallow-water model}

The numerical model to be analyzed in sections 3 and 4 is a global semi-Lagrangian finite-element model of the shallow-water equations (Côté et al. 1993). Owing to its inherent dissipation, due primarily to the spatial interpolation required by the semi-Lagrangian scheme (McCalpin 1988), the model can be run without explicit diffusion.

The basic state is chosen following Polvani et al. (1995), who used a jet with a maximum speed of $50 \mathrm{~m}$ $\mathrm{s}^{-1}$ at $50^{\circ} \mathrm{N}$, a zero wind line at $23^{\circ} \mathrm{N}$, and weak easterlies at the equator and in the summer (i.e., southern) hemisphere. The expression

\footnotetext{
${ }^{2}$ For the purposes of this work a fully turbulent flow is taken to be one in which the velocity field is both spatially and temporally irregular.
} 


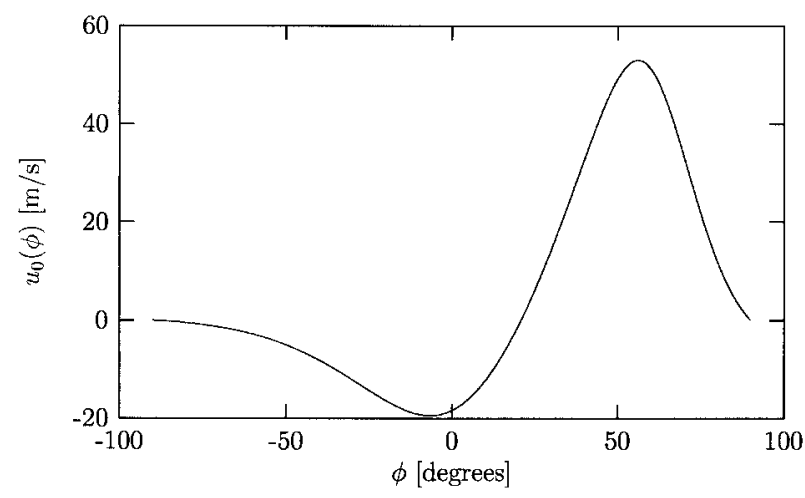

FIG. 1. Initial zonal velocity profile for the standard jet, Eq. (2).

$u(\phi)=2 A \cos \phi \operatorname{sech}\left(\frac{\phi-a_{1}}{a_{2}}\right)-2 B \cos \phi \operatorname{sech}\left(\frac{\phi}{b_{1}}\right)$,

with

$$
\begin{array}{ll}
A=55 \mathrm{~m} \mathrm{~s}^{-1}, & a_{1}=1.1, \quad a_{2}=0.3, \\
B=12 \mathrm{~m} \mathrm{~s}^{-1}, & b_{1}=0.5,
\end{array}
$$

yields a jet with maximum speed of $53 \mathrm{~m} \mathrm{~s}^{-1}$ at $56^{\circ} \mathrm{N}$ and zero wind line at $21^{\circ} \mathrm{N}$ (Fig. 1). Except for section $3 \mathrm{~b}$, these parameters are used for all the simulations described below. The initial height field is obtained from gradient wind balance, that is,

$$
h(\phi)=h_{0}-\int^{\phi^{\prime}} a u\left(\phi^{\prime}\right)\left[f+\frac{\tan \phi^{\prime}}{a} u\left(\phi^{\prime}\right)\right] d \phi^{\prime},
$$

where $h_{0}=9.5 \mathrm{~km}$ is the mean depth of the unperturbed fluid layer, $a$ is the radius of the earth, and $f$ is the Coriolis parameter.

As is by now standard, a forcing is added to the height field in order to simulate the dynamics of a perturbed polar vortex. The governing equations are (e.g., Juckes 1989)

$\frac{\partial \zeta_{a}}{\partial t}=-\nabla \cdot\left(\mathbf{u} \zeta_{a}\right)$

$\frac{\partial D}{\partial t}=(\mathbf{k} \times \boldsymbol{\nabla}) \cdot\left(\mathbf{u} \zeta_{a}\right)-\nabla^{2}\left[\gamma^{-1}\left(h+h_{f}\right)+\frac{1}{2}\left(u^{2}+v^{2}\right)\right]$,

$\frac{\partial h}{\partial t}=-\nabla \cdot(h \mathbf{u})$,

where $\zeta_{a}$ is the absolute vorticity, $D$ is the divergence, $h$ is the fluid height, $h_{f}$ is the forcing amplitude, and $\gamma$ $=a^{2} \Omega^{2} / g h_{0}$ is the rotational Froude number. Following Salby et al. (1990a) and Salby (1992), the forcing takes the form

$$
h_{f}=\frac{z_{0}}{2} A(t) B(\phi) C(\lambda, t),
$$

with

$$
\begin{aligned}
A(t) & =\frac{1}{2}\left[1+\tanh \frac{t-5}{2.5}\right], \\
B(\phi) & =B_{0} \cos \phi \exp \left\{-\left[\left(\phi-\phi_{0}\right) / \Phi\right]^{2}\right\}, \\
C(\lambda, t) & =z_{s}[1+\cos \lambda]+z_{t}[\cos (\lambda-c t)] .
\end{aligned}
$$

The time envelope $A(t)$ reaches its maximum value of unity at approximately 10 days; the latitudinal envelope $B(\phi)$ has parameters $\phi_{0}=75^{\circ}, \Phi=30^{\circ}$, and $B_{0}^{-1}=$ 0.39 (so that the maximum value of $B(\phi)$ is unity); the zonal structure function $C(\lambda, t)$ determines the nature of the planetary wave forcing $\left(z_{s}\right.$ and $z_{t}$ denote the stationary and transient amplitudes, $c$ is the phase speed of the transient).

All the results in this paper were obtained using a horizontal grid of $144 \times 72\left(2.5^{\circ} \times 2.5^{\circ}\right)$ and a time step of $900 \mathrm{~s}$ for the dynamical fields; the results are similar for increased spatial and temporal resolution. ${ }^{3}$ Winds are output every $\Delta t_{\mathrm{sw}}=3 \mathrm{~h}$. As the vorticity is a diagnostic variable in the model the shallow-water potential vorticity is computed from the velocity and height fields, that is, $q=\{f+[\partial v / \partial \lambda-\partial(u \cos \phi) /$ $\partial \phi] / \cos \phi\} / h$.

\section{Tracer fields}

In this section qualitative evidence for the spatial organization of the tracer field is presented. High-resolution tracer fields are constructed from offline particle integrations and used to demonstrate the existence of secondary cat's eyes. Previous studies (e.g., Polvani et al. 1995) have emphasized transport across the polar and subtropical edges of the surf zone rather than the structure of the surf zone per se.

The particle advection calculations are carried out by applying linear interpolation in space and time to wind fields from the shallow-water model. The calculations are done in spherical polar coordinates, that is,

$$
\frac{D \lambda}{D t}=u /(a \cos \phi), \quad \frac{D \phi}{D t}=v / a,
$$

where $\lambda$ and $\phi$ are the longitude and latitude, respectively, of a tracer particle. The use of constant $a$ (the height of the fluid layer is ignored) entails negligible error; there have been no problems with the singularity at the poles. A fourth-order Runge-Kutta scheme with a time step $\Delta t_{\mathrm{RK}}=225 \mathrm{~s}$ is used. The results presented are generally insensitive to $\Delta t_{\mathrm{RK}}$ and $\Delta t_{\mathrm{SW}}$ (see section $3 \mathrm{e}$, however). Unless otherwise stated, the initial particle distribution consists of approximately 70000 particles uniformly spaced from $15.5^{\circ}$ to $52.5^{\circ} \mathrm{N}$ at $0.5^{\circ}$ intervals and from $0.2^{\circ}$ to $359.8^{\circ} \mathrm{E}$ at $0.32^{\circ}$ intervals, which provides an approximate cover of the surf zone

\footnotetext{
${ }^{3}$ The quantitative effect of increased spatial resolution is considered in Part II.
} 
for $z_{0}=1000 \mathrm{~m}$. (In sections $3 \mathrm{a}$ and $3 \mathrm{~b}$ we extend this distribution so as to resolve the structure of the surf zone more clearly.)

\section{a. Stationary forcing}

We begin with stationary forcing. Figure 2 shows the tracer field at 20,30 and 50 days for $z_{0}=1000 \mathrm{~m}$. The evolution in this figure resembles that seen in analytical solutions from Rossby wave critical-layer theory (e.g., Fig. 2 of Killworth and McIntyre 1985) and in criticallayer simulations (Haynes 1989). In Fig. 2a one sees the "tracer isolines" beginning to wrap up slightly north of the zero wind line (cf. Randel and Held 1991). In Figs. 2b and 2c a well-defined critical layer emerges: a cat's eye, where there is much filamentation and wrapup, is bounded by unbroken isolines. (Note that because the primary cat's eye is tilted, periodic boundary conditions give the false impression of two distinct primary cat's eyes, especially in Fig. 2a.)

The tracer field produced by the offline integration is consistent with the potential vorticity field (Fig. 3). As in contour advection, much more small-scale structure is present in the offline integration; however, in the present case, the structure of the surf zone may be appreciated more readily, contour advection calculations being restricted to only a few contours (albeit very finely resolved). This enables one to establish connections with standard analyses of chaotic advection, which are rooted in the geometric structure of the model in question.

Secondary cat's eyes are visible in Figs. $2 \mathrm{~b}$ and 2c, as well as in Fig. 3. Salby et al. (1990b) observed the formation of "rolls" of potential vorticity in the critical layer when their equivalent barotropic model was run without numerical viscosity. Such secondary cat's eyes are a generic feature of critical-layer simulations, where they arise from barotropic instability of the primary cat's eye (Haynes 1989). They are seen in numerical simulations of wave breaking induced by isolated Rossby wave trains (Held and Phillips 1987). There are also hints of them in stratospheric $\mathrm{N}_{2} \mathrm{O}$ tracer fields calculated using reverse domain filling (Sutton et al. 1994), though a polar stereographic projection does hinder identification in this case.

It is interesting to note that the potential vorticity is approximately homogenized within the cat's eyes. This suggests that the PV in the critical layer is indeed being mixed as if it were a passive tracer, as is assumed in the study of Ngan and Shepherd (1997).

The existence of cat's eyes is significant because it is prima facie evidence of large-scale structure in the velocity field, a prerequisite for the applicability of chaotic advection (see the remarks in the introduction). In the context of chaotic systems it is often said that the heteroclinic structure is a template for chaotic dynamics (e.g., Beigie et al. 1994); here it would appear that the cat's eyes function as a time-dependent organizing structure for mixing and transport in the surf zone.

The picture for other values of $z_{0}$ is similar as long as $z_{0}$ does not exceed moderate amplitudes. In Figs. $4 \mathrm{a}$ and $4 \mathrm{~b}$ the cat's eye structure may be easily discerned, though the degree of coarse-grain homogenization in the tracer field varies with $z_{0}$. For large $z_{0}$, however, the vortex splits and only fragments of the cat's eye structure remain (Fig. $4 \mathrm{c})$.

\section{b. Basic-state shear}

Previous studies of stratospheric mixing and transport have used "realistic" basic states, that is, jets with maximum velocities around $50 \mathrm{~m} \mathrm{~s}^{-1}$ at $50^{\circ} \mathrm{N}$ and zero wind lines in the Tropics $\left(20^{\circ} \mathrm{N}\right.$ or thereabouts). As this is also the case in the preceding section, it is important to determine how (and to what extent) the results described above depend on the basic state.

For simplicity we consider two representative cases: a jet whose initial zonal velocity is half that of the standard jet, ${ }^{4}$ and another whose initial zonal velocity is twice as large. ${ }^{5}$ The tracer field at 50 days for both cases is shown in Fig. 5.

From this figure it would appear that the cat's eyes are robust with respect to changes in the basic state. This is the case in Rossby wave critical-layer theory: changes in the basic-state shear affect the width of the critical layer, not its formation. Although critical-layer theory is not strictly applicable to these experiments, the width of the cat's eyes does increase as the (basic state) shear decreases, in accordance with theory.

It will also be noticed that the wavenumber of the characteristic cat's eye structure is affected by the background shear. In numerical simulations of the barotropic critical-layer equations (Haynes 1989), the nature of the instability of the primary cat's eye is governed by the aspect ratio $\mu$ : for $\mu \rightarrow 0$ [the Stewartson-Warn-Warn (SWW) limit] the instability occurs at small scales and the cat's eye structure is essentially destroyed; for finite $\mu$, the small-scale instability is suppressed (cf. Dritschel et al. 1991), and secondary cat's eyes appear. The value of $\mu$ provides a useful guide to the interpretation of the present results. For the standard jet profile (2), $\mu \sim 1 / 4$ and secondary cat's eyes appear. In the critical-layer simulations, such a value of $\mu$ also yields secondary cat's eyes. Likewise, the instability is stronger when shear is halved ( $\mu$ reduced) and weaker when it is doubled ( $\mu$ increased).

Shear instability of the nonlinear Rossby wave critical layer is not a hindrance to the applicability of chaotic advection. For realistic stratospheric flows $\mu$ is not infinitesimally small and one does not see the kind of

\footnotetext{
${ }^{4}$ The parameters are $A=27.5, B=6, a_{1}=1.1, a_{2}=0.3$, and $b_{1}$ $=0.5 \mathrm{~m} \mathrm{~s}^{-1}$ : only the amplitudes in (2) are changed.

${ }^{5}$ Here $A=110, B=24 \mathrm{~m} \mathrm{~s}^{-1}$.
} 
(a)

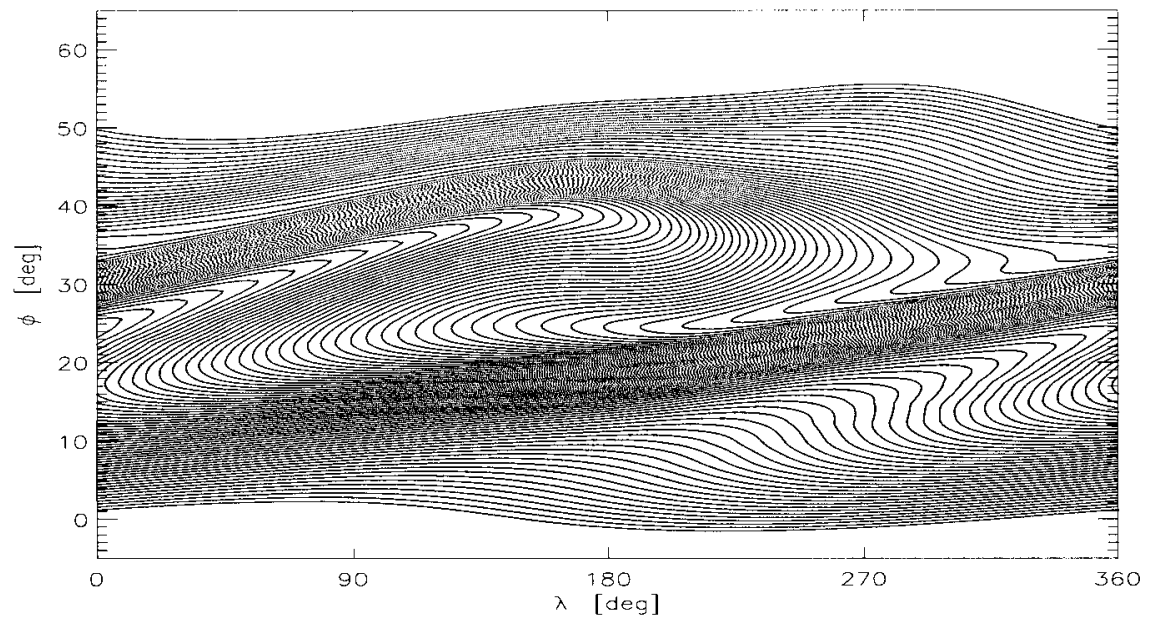

(b)

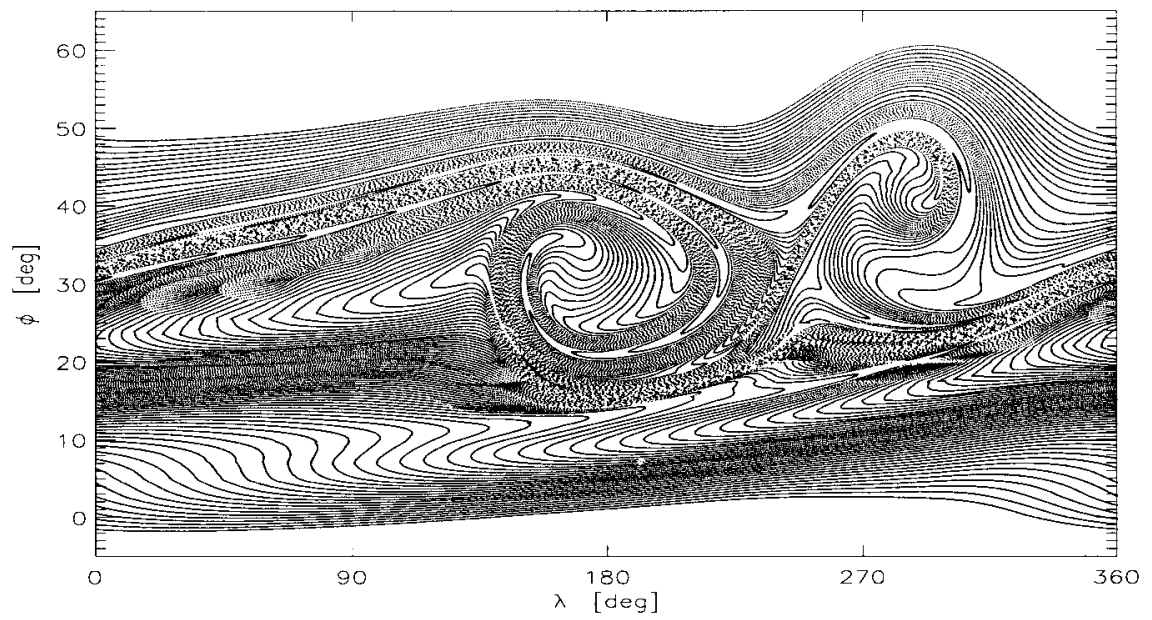

(c)

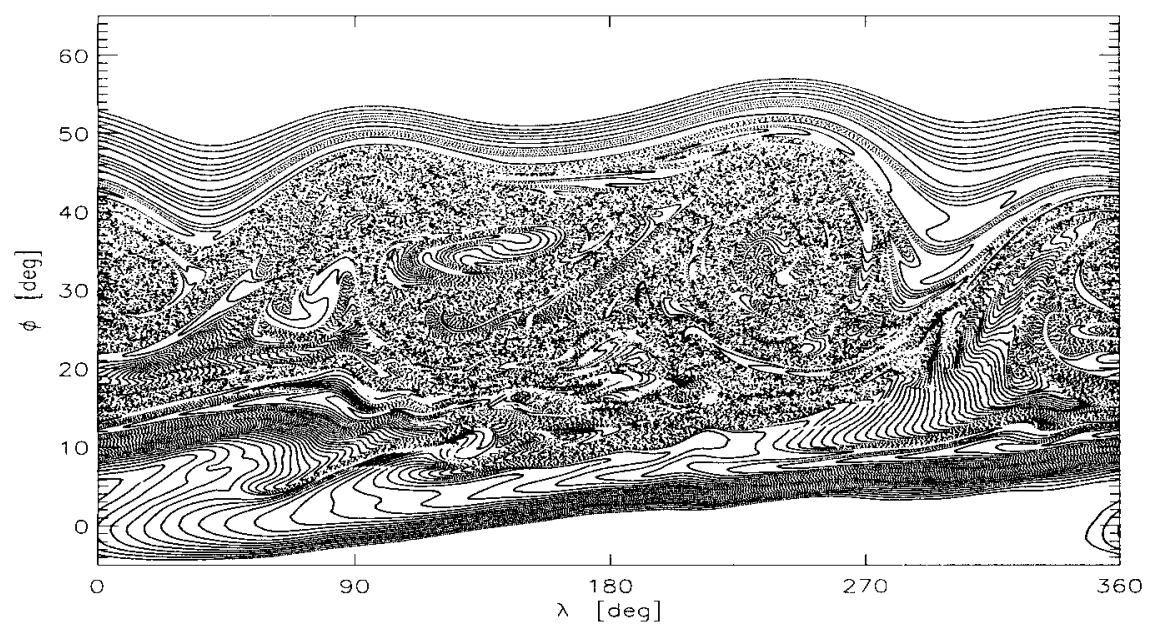

FIG. 2. Tracer fields for moderately strong stationary forcing, $z_{0}=1000 \mathrm{~m}$. (a) 20 days; (b) 30 days; (c) 50 days. The initial tracer distribution lies along latitude circles and $\phi$ is extended down to $0.5^{\circ}$. 


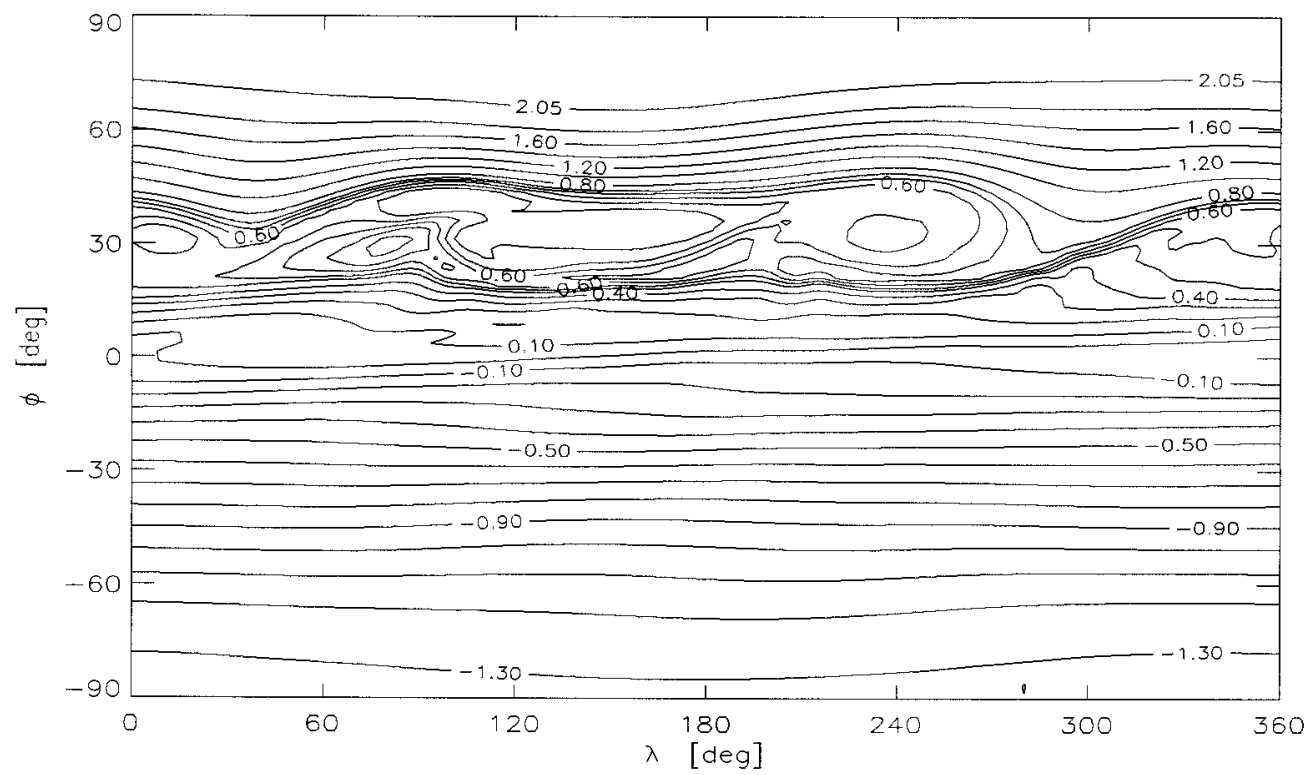

FIG. 3. Potential vorticity at 50 days for $z_{0}=1000 \mathrm{~m}$. The projection is regular latitude-longitude. Contour labels are in units of $10^{-8} \mathrm{~m}^{-1} \mathrm{~s}^{-1}$; the contour spacing is irregular.

violent instability that is observed in the SWW limit. The instability is an intrinsic feature of the critical-layer dynamics, and it makes no sense-nor is there any need-to disentangle it from the "normal" critical-layer evolution. For flows representative of the stratosphere, the main effect of the instability is to change the morphology of the cat's eye; it is not strong enough to change the essential fact that small-scale tracer structure is determined by the large-scale flow.

It is also worth mentioning in passing that coarsegrain homogenization is enhanced when the basic-state shear is increased. This is because the advective timescale decreases; equivalently, velocity gradients increase. These effects may be quantified by computing finite-time Liapunov exponents (see Part II, section 4).

\section{c. Transient forcing}

The critical line of a wave-1 travelling wave is found where its ground speed at a given latitude-given by $c$ $=2 \pi a \cos \phi / T, T$ being the period of the wave-matches the basic-state velocity. Qualitative and quantitative effects of wave transience have been considered previously by Salby (1992) and Bowman (1993b), with the emphasis once more on transport into and out of the polar vortex.

The results are generally as expected. For purely transient forcing with $c>0$, the surf zone is shifted to the north and the shear instability is somewhat weaker than for the stationary case, the shear being stronger at the zero wind line of the transient. [A related phenomenon is that sheared disturbances, which are ubiquitous in the outer region associated with a forced Rossby wave critical layer (e.g., Haynes 1987), are more prominent.] For combined stationary and transient forcing the picture is also similar: unlike the case for idealized time-periodic chaotic advection models, which retain only a single temporal harmonic (e.g., Ngan and Shepherd 1997), stationary-transient interactions do not lead to markedly different results. This is because stationary forcing in the shallow-water system, or for that matter in a barotropic system, already generates complicated time dependences in the velocity field (see section 4a): explicit transient forcing is not required.

\section{d. Deformation radius}

In the runs described above the initial mean fluid depth is $h_{0}=9.5 \mathrm{~km}$. This value is consistent with those used in previous shallow-water studies $\left(h_{0} \sim 8 \mathrm{~km}\right)$. It is interesting to vary $h_{0}$ because this provides one way of assessing the importance of the deformation radius $L_{R}$. To isolate the effect of the deformation radius it is necessary to scale the perturbation amplitude with $h_{0}$. [Recall that the forcing amplitude $h_{f}$ enters the equations of motion (3) through a term $h+h_{f}$ in the divergence equation.]

Once again the cat's eye structure is a robust feature. The width of the surf zone does, however, increase slightly with $h_{0}$; as in section $3 \mathrm{~b}$, this is consistent with Rossby wave critical-layer theory. Although the basicstate shear is fixed, the potential vorticity gradient decreases with increasing $h_{0}$, which leads to a wider critical layer.

One might expect the tracer field to be more sensitive to $L_{R}$ since vortex interactions should strengthen as $L_{R}$ increases and the flow becomes more barotropic (cf. Waugh and Dritschel 1991). This is not, evidently, an 
(a)

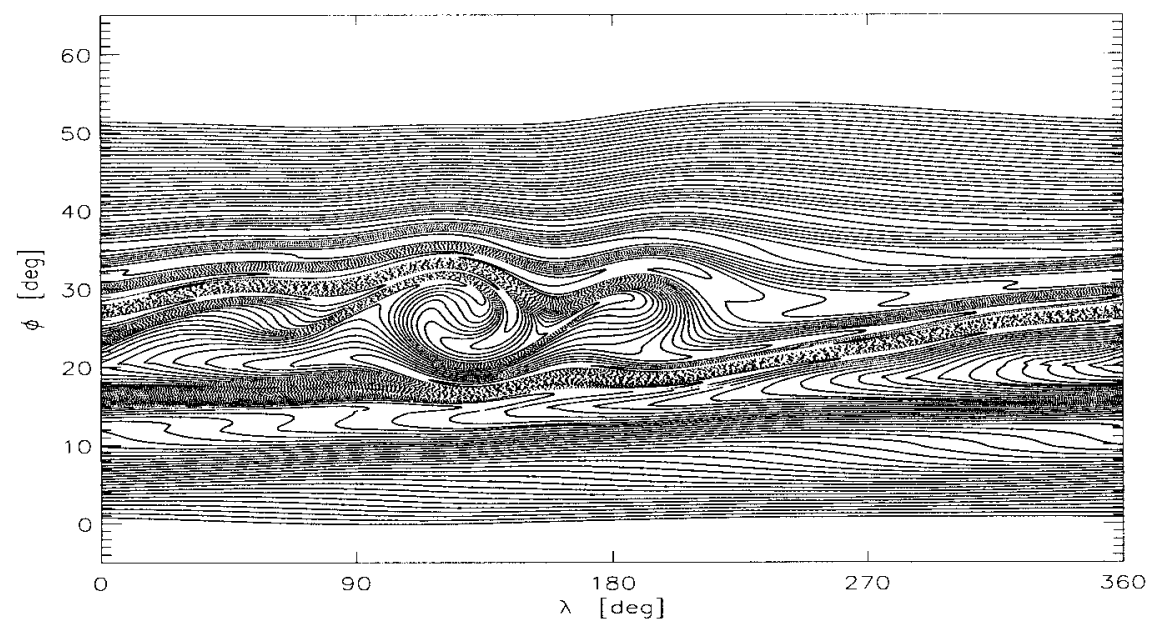

(b)

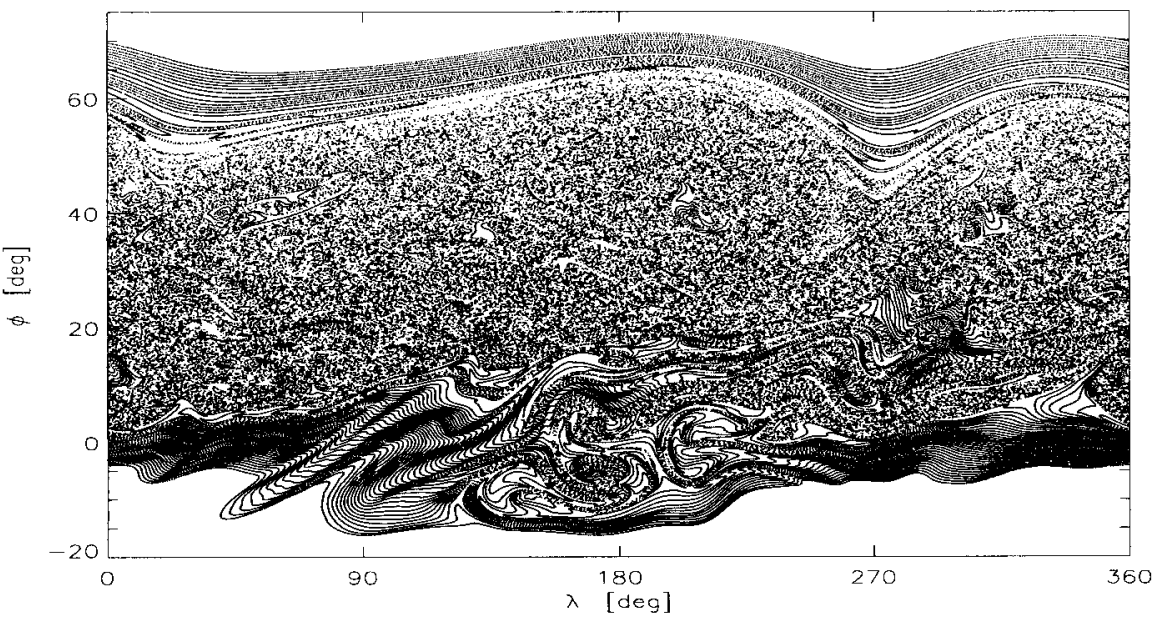

(c)

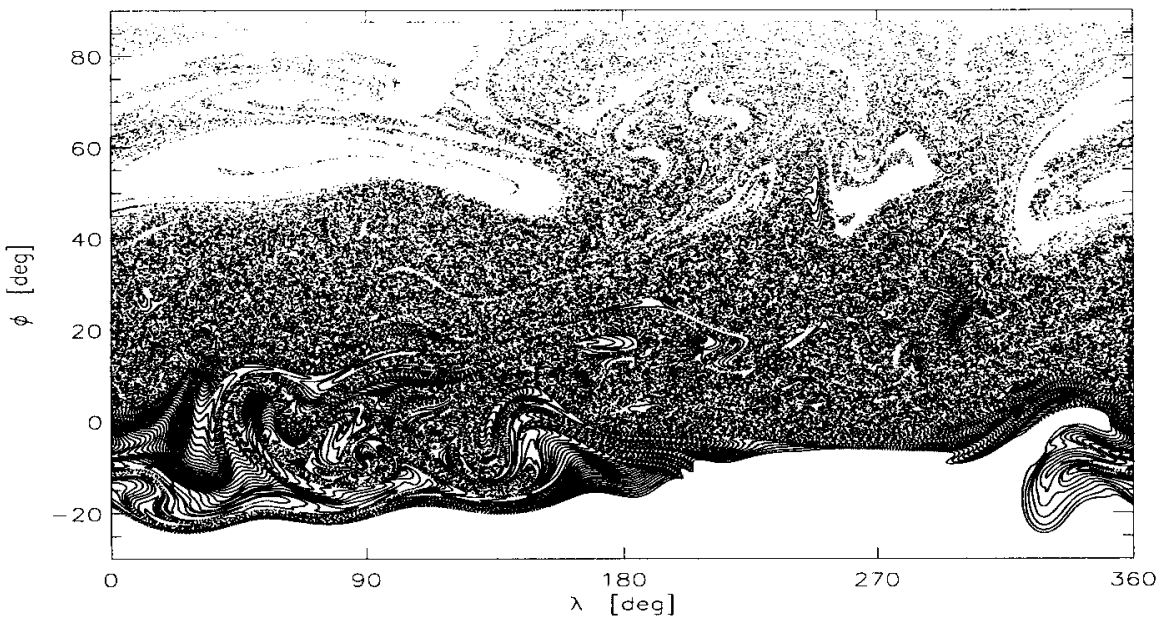

FIG. 4. Tracer field at 50 days for different values of the stationary forcing amplitude, $z_{0}$. (a) $z_{0}=$ $250 \mathrm{~m}, \phi \in\left[0.5^{\circ}, 52.5^{\circ}\right]$; (b) $z_{0}=2000 \mathrm{~m}, \phi \in\left[-10^{\circ}, 65^{\circ}\right]$; (c) $z_{0}=3000 \mathrm{~m}, \phi \in\left[-15^{\circ}, 65^{\circ}\right]$. Note the change of scale between panels. 
(a)

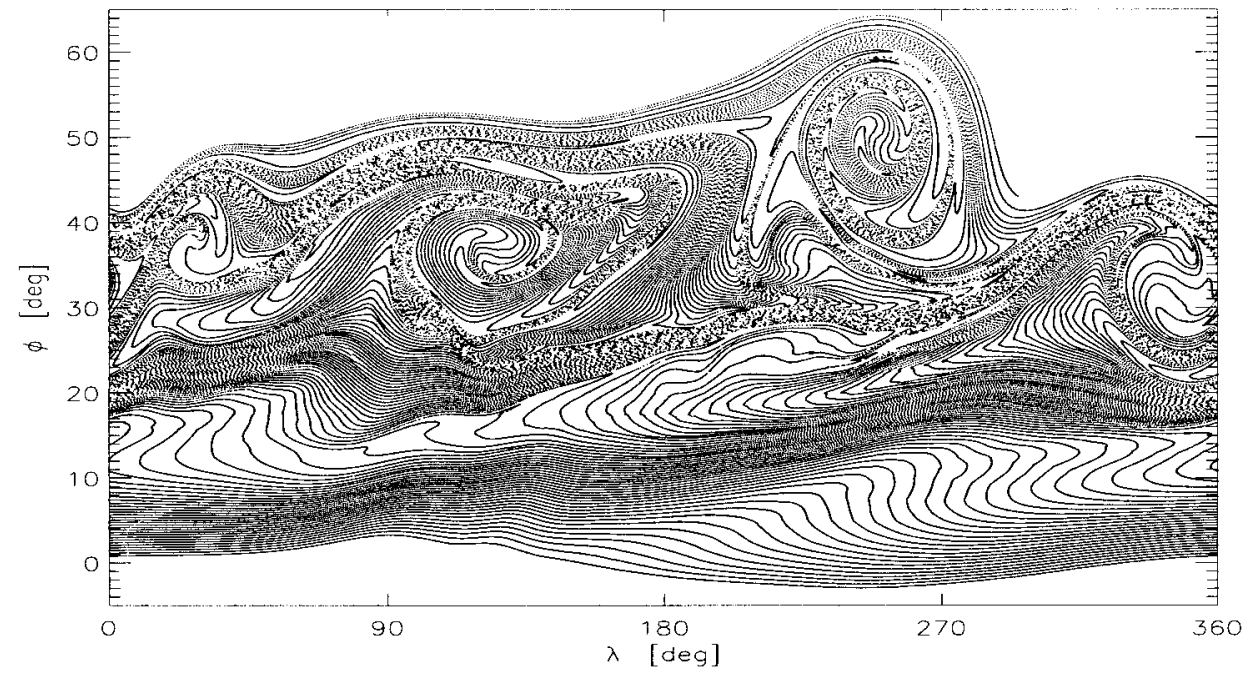

(b)

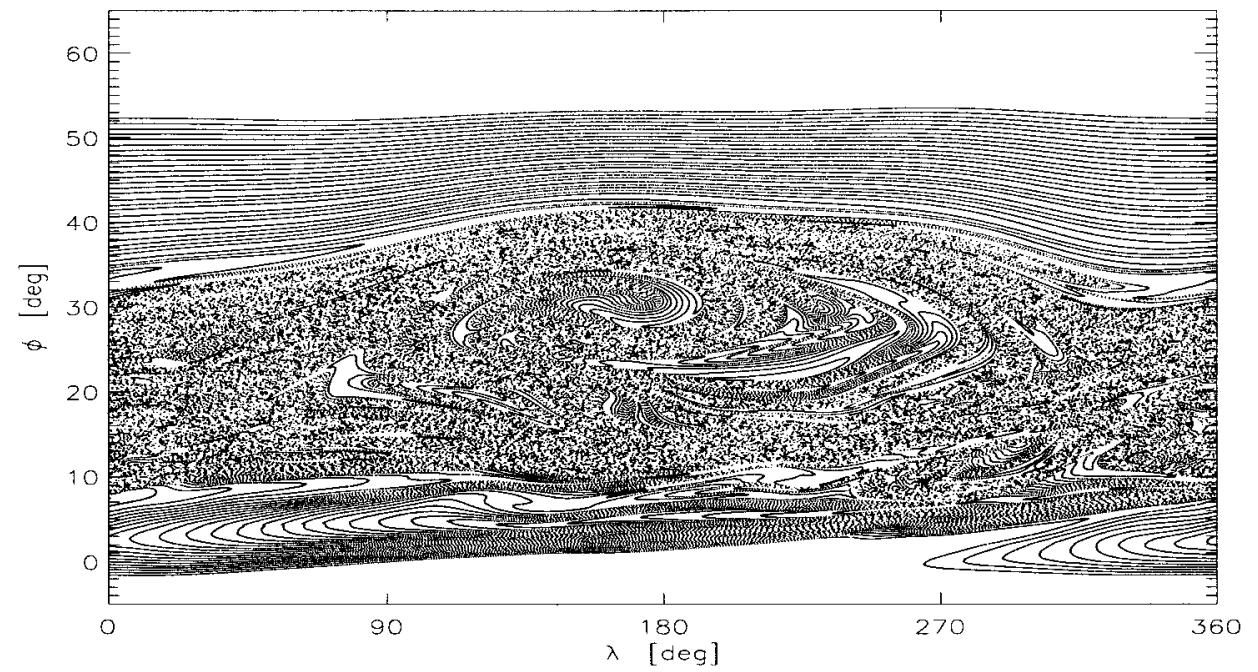

FIG. 5. Tracer field at 50 days for different basic states. The initial velocity is scaled by (a) $0.5 \times$ and (b) 2 $\times$ relative to the standard profile, Eq. (2). The initial distributions have $\phi \in\left[0^{\circ}, 60^{\circ}\right]$ and $\phi \in\left[0.5^{\circ}, 52.5^{\circ}\right]$, respectively; $z_{0}=1000 \mathrm{~m}$.

important consideration here because the variation in $L_{R}$ is limited.

\section{e. Stochastic forcing}

The preceding sections have shown that the formation of a cat's eye structure in the surf zone is robust when a spatially coherent large-scale perturbation is applied. We now consider the effect of random, small-scale fluctuations by introducing stochastic forcing into the velocity and height fields:

$$
F(\lambda, \phi, t) \rightarrow F(\lambda, \phi, t)\left[1+\delta_{F} \eta\right],
$$

where $F$ stands for a velocity component or the height perturbation (i.e., $h_{f}$ ), and $\eta \in[-1,1]$ is a white-noise random variable (in space and time). Stochastic forcing is of interest because while the large-scale velocity fields obtained from numerical models or meteorological analyses (e.g., Bowman 1993a; Waugh and Plumb 1994; Polvani et al. 1995) may be representative of stratospheric flow, discrepancies at small scales are inevitable. Stochastic forcing may also be regarded as a crude surrogate for gravity wave effects (see section 5).

We begin with pulse forcing of the velocity field. A random pulse of amplitude $\delta_{v}=0.1$ is added at the 


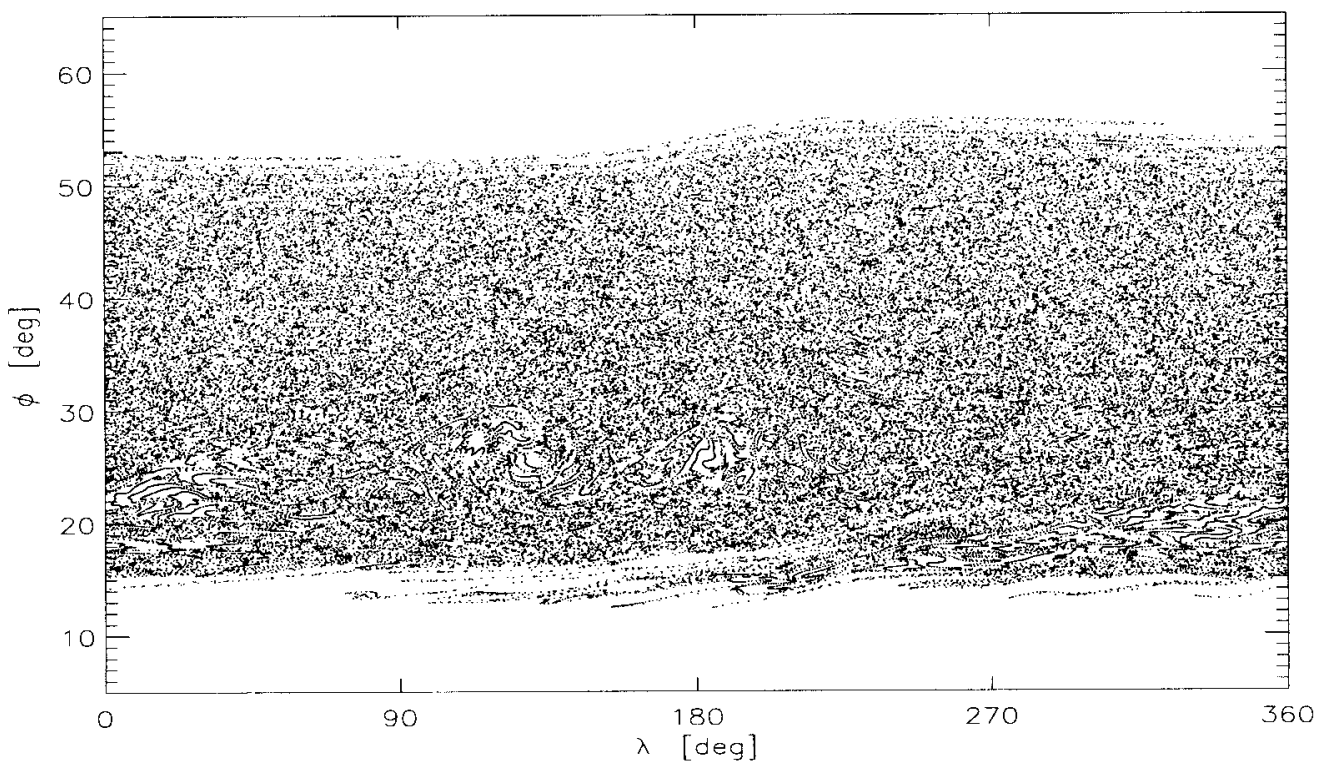

FIG. 6. Tracer field at 50 days for a pulse to the velocity field of amplitude $\delta_{v}=0.1$ applied at time $t=0 . z_{0}$ $=250 \mathrm{~m}$.

initial time step. As with stochasticity in idealized chaotic advection models (Ngan and Shepherd 1997), particle trajectories are smeared out by the addition of stochasticity to the velocity field (Fig. 6). Nevertheless, there is a hint of the geometrical structure of the unperturbed velocity field: multiple cat's eyes are just visible (cf. Fig. 4a). From Fig. 7 one sees that the pulse has a minimal effect on the PV field: the cat's eye structure is in fact very well defined; spatial organization of the flow persists. In contrast with the behavior of the barotropic Rossby wave critical layer (Haynes 1989), the time evolution of the PV field is relatively insensitive to the addition of noise at the initial time step; shear instability in the shallow-water system is relatively weak, as discussed in section $3 \mathrm{~b}$. The tracer and PV fields differ considerably in appearance because the "scrambling" of the tracer field caused by the pulse persists even at long times, when the dynamical effect of the pulse has worn off; the tracer and PV fields remain decorrelated.

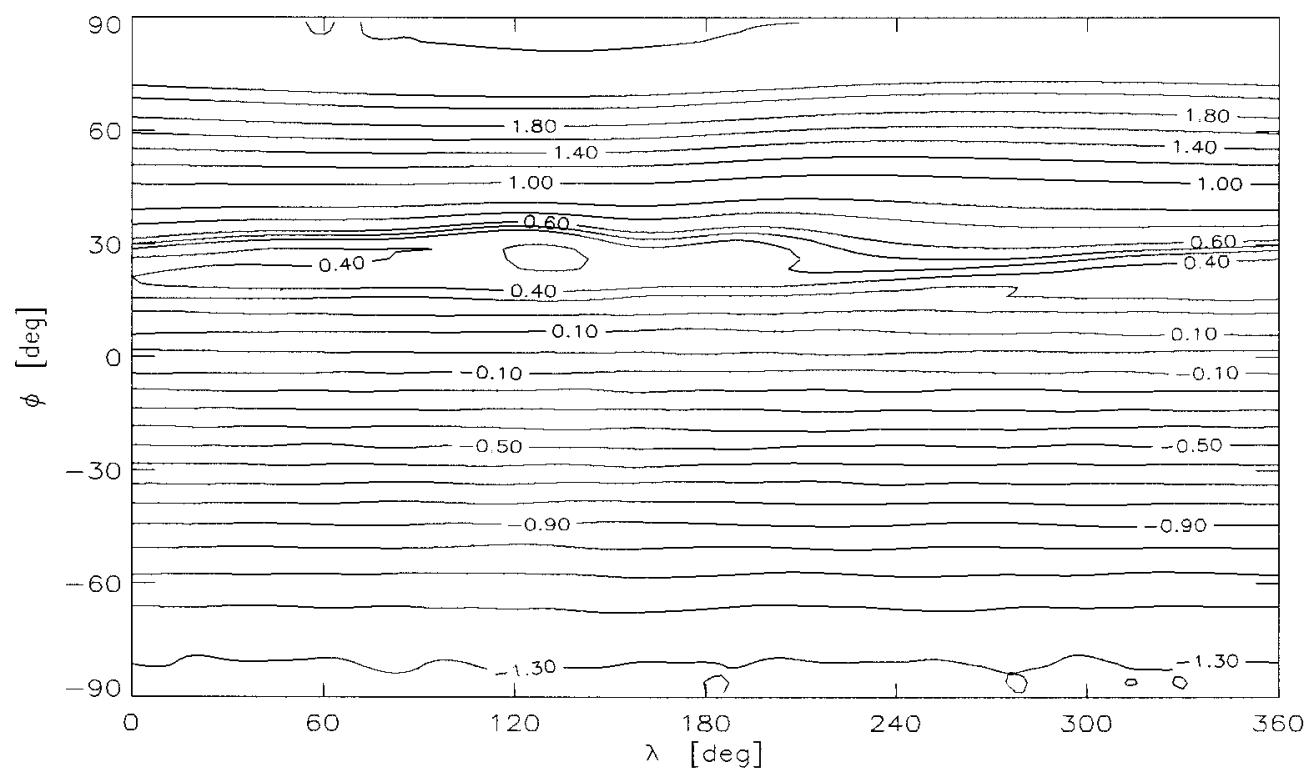

FIG. 7. Potential vorticity at 50 days for $z_{0}=250 \mathrm{~m}$ and a velocity pulse of amplitude $\delta_{v}=0.1$. Contour labels are in units of $10^{-8} \mathrm{~m}^{-1} \mathrm{~s}^{-1}$; the contour spacing is irregular. 


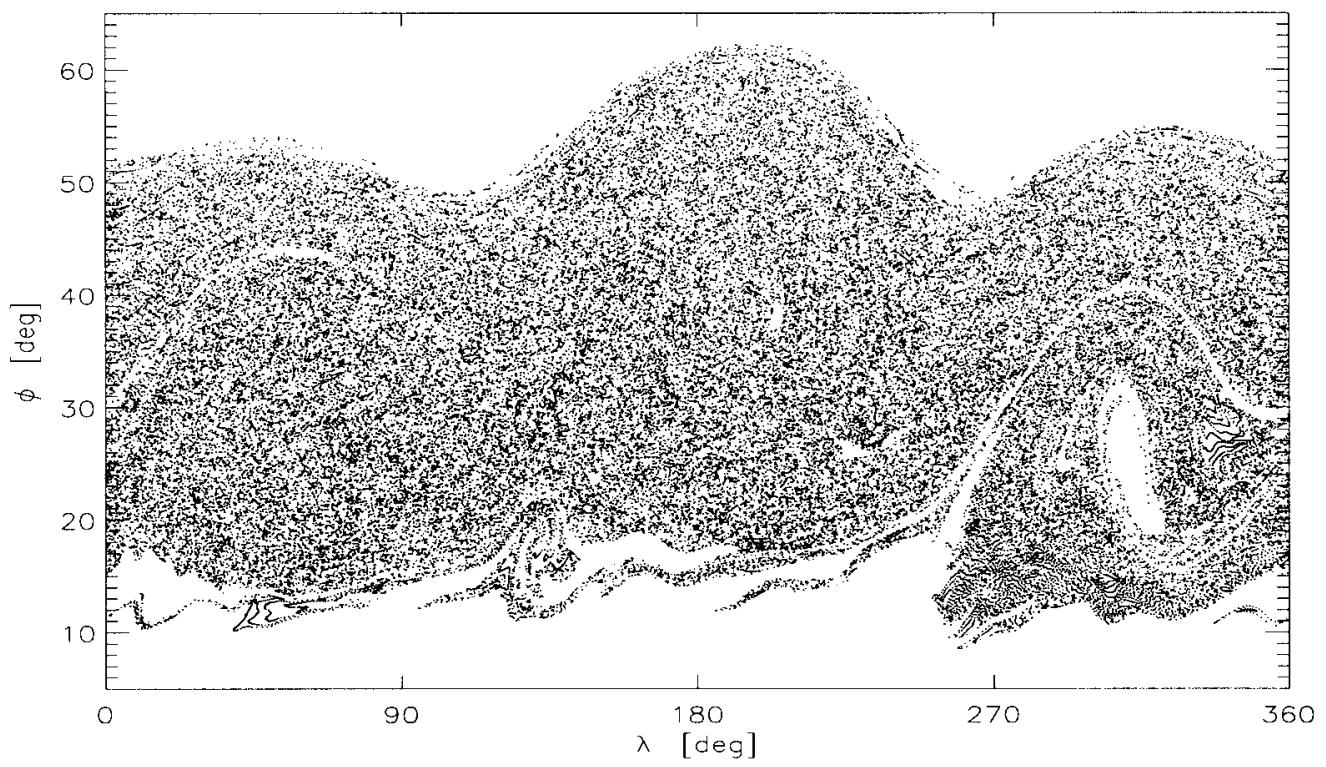

FIG. 8. Tracer field at 50 days for continuous stochastic forcing. $\delta_{v}=0.002, z_{0}=1000 \mathrm{~m}$.

Continuous stochastic forcing is now considered. For brevity we consider only perturbations to the velocity field; stochasticity in the height field generally has a smaller effect. For moderate noise amplitudes (Fig. 8), the cat's eye structure persists, as in the random pulse case above: once more the main effect of stochasticity is to smear out particle trajectories; there is only a slight effect on the corresponding PV fields.

This behavior is easily understood. For small noise amplitudes the particle advection equations may be written in Langevin form, that is,

$$
\dot{x}=u(x, y, t)+\tilde{\delta}_{u} \eta, \quad \dot{y}=v(x, y, t)+\tilde{\delta}_{v} \eta,
$$

where $\eta$ is a Gaussian white-noise variable and $\tilde{\delta}_{u}, \tilde{\delta}_{v}$ $\ll 1$ are noise amplitudes. Consequently the stochasticity acts simply as an effective diffusivity on the particle trajectories (e.g., Crisanti and Vulpiani 1993); there is still spatial organization of the large-scale flow-the cat's eye structure persists-and as with the cases without stochastic forcing, chaotic advection occurs.

The situation is different for large amplitudes, however. When the noise amplitude is large, nonlinear interactions are important and the structure of the velocity field may be altered. To see this consider Fig. 9, which shows the potential vorticity field at 50 days for three different values of $\delta_{v}$. For the largest value of $\delta_{v}$ a cat's eye structure no longer exists; the critical layer is essentially destroyed by stochasticity, in agreement with the findings of Salby (1992). This suggests that chaotic advection does not occur when there is sufficiently strong stochastic forcing (cf. section 4a). By contrast, the spatial structure of idealized Hamiltonian models, such as are utilized in chaotic advection studies, is unaffected by stochasticity (Ngan and Shepherd 1997) since nonlinear interactions are excluded.
It is important to note that in cases with continuous stochastic forcing potential vorticity is not conserved: the PV and tracer are uncorrelated at all times. This is in contrast to the deterministic cases described above (e.g., section 3a), where, in principle, the PV and tracer fields are equivalent. (In practice they differ because of numerical effects.) Here the transport and mixing of the PV and tracer fields is even in principle somewhat different. For weak stochasticity, however, the persistence of the cat's eye structure suggests that the same mechanism is operative: chaotic advection by the large-scale velocity field.

Despite the highly disordered appearance of the flows depicted above, these results do appear to be robust. Quantitative diagnostics such as dispersion statistics and Liapunov exponents scale smoothly with $\delta_{v}$ (see Part II): they do not assume unphysical values, though they are dependent on spatial resolution.

\section{Correlation times}

The results of the preceding section demonstrate that there is spatial organization of the velocity field in the shallow-water system if stationary forcing does not exceed moderate amplitudes and if stochastic forcing is sufficiently weak. We now consider whether this velocity field is quasi-regular, in the sense of our introductory remarks.

Correlation times of the velocity derivatives are computed in order to provide quantitative evidence for chaotic advection. As mentioned in the introduction, chaotic advection may be thought of as a situation in which the Lagrangian correlation time is short compared to the Eulerian correlation time (Swanson and Pierrehumbert 
(a)

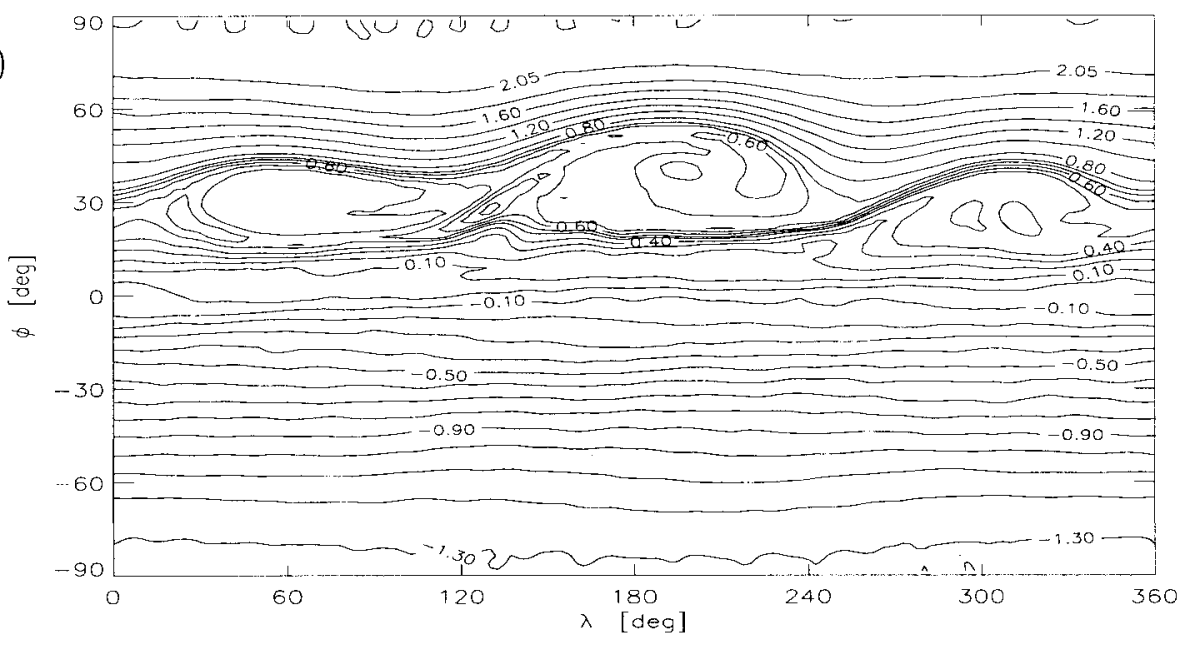

(b)

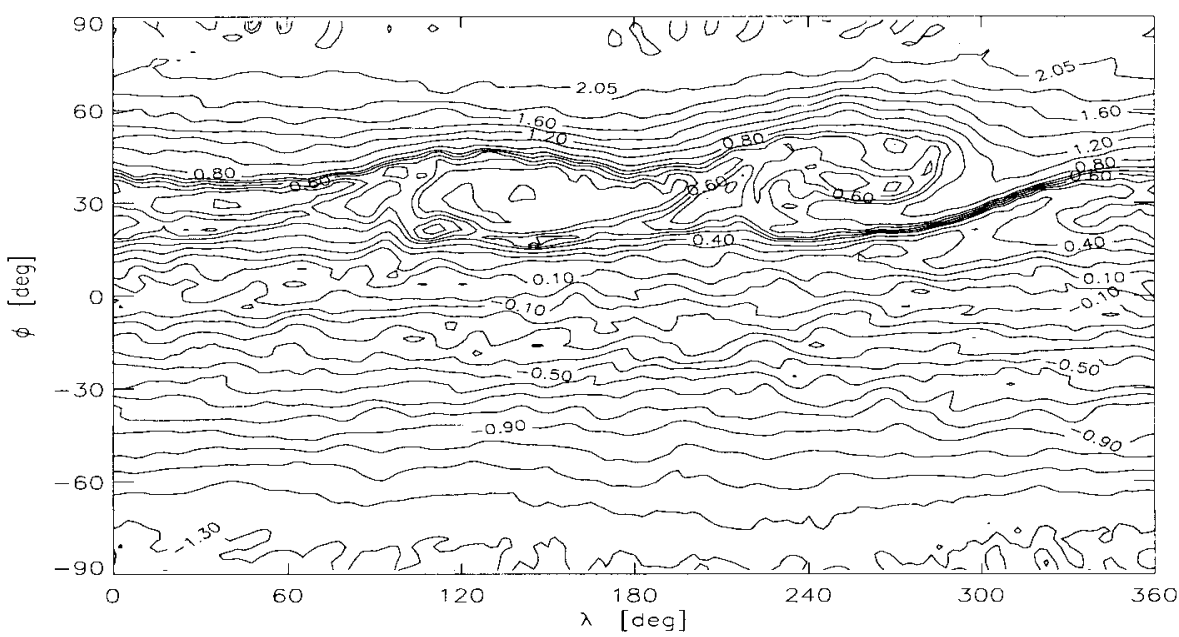

(c)

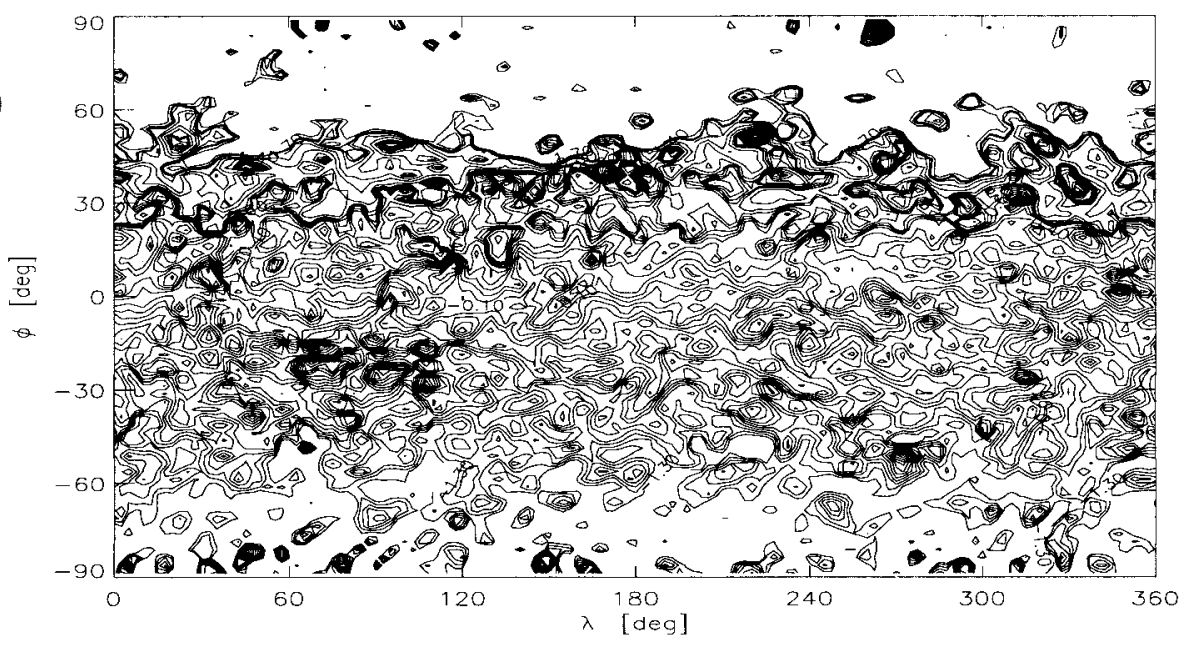

FIG. 9. Potential vorticity field at 50 days for $z_{0}=1000 \mathrm{~m}$ and increasing stochasticity. (a) $\delta_{v}=0.002$;

(b) $\delta_{v}=0.01$; (c) $\delta_{v}=0.1$. Contour labels are in units of $10^{-8} \mathrm{~m}^{-1} \mathrm{~s}^{-1}$; the contour spacing is irregular. 
1997). The correlation times may be inferred from the autocorrelations of the velocity derivatives:

$$
r(k)=\frac{\frac{1}{N-k} \sum_{i=1}^{N-k}\left(u_{i}-\bar{u}\right)\left(u_{i+k}-\bar{u}\right)}{\frac{1}{N} \sum_{i=1}^{N}\left(u_{i}-\bar{u}\right)^{2}},
$$

where $\left\{u_{i}\right\}, i=1, \ldots, N$ is a time series. For convenience the autocorrelations are shown as a function of a dimensional time $\tau^{\prime}\left(\tau^{\prime}=k \Delta t_{\mathrm{RK}}\right)$; the Lagrangian autocorrelation is denoted by $r^{L}$.

Although they are qualitatively similar, autocorrelations of the velocity derivatives rather than the velocities themselves are shown so as to facilitate comparison with random-strain theory. In random-strain theory (e.g., Batchelor 1959; Kraichnan 1974; Chertkov et al. 1995), which is sometimes taken to be an idealized model of turbulent mixing, it is assumed that the velocity derivatives are zero-mean, random functions with constant correlation time. Recently random-strain theory has been invoked to help explain the vertical-scale cascade of tracer in the lower stratosphere (Haynes and Anglade 1997; see also Juckes and McIntyre 1987).

The work of Pierrehumbert (1994) and Antonsen et al. (1996) suggests that there is a close connection between random-strain theory and chaotic advection. Despite its connections with turbulent mixing, randomstrain theory is of fairly wide applicability. Basically, all that is required is that the velocity derivatives along particle trajectories be random functions of time so that the stretching of material lines can be described in terms of a mean strain and the correlation time of the derivatives, or, alternatively, that a large-scale (Lagrangian) strain field be defined in a statistical sense (cf. Kraichnan 1974). One expects these conditions to be met when there is chaotic advection. Yet chaotic advection and random strain are not necessarily equivalent, for the latter is a purely statistical concept. With this distinction in mind, we therefore introduce the concept of Lagrangian random strain, a situation in which the velocity derivatives decorrelate along particle trajectories but not at fixed spatial locations. Lagrangian random strain (i.e., temporal quasi-regularity) is a prerequisite for chaotic advection.

For periodic or quasiperiodic chaotic advection models, the Eulerian correlation time is, by construction, infinite. Aperiodicity will presumably lead to finite Eulerian correlation times, but this is not necessarily inconsistent with the presence of chaotic advection (cf. Malhotra and Wiggins 1998).

\section{a. Eulerian correlation times}

Autocorrelations of the four spatial derivatives, $u_{x}$, $u_{y}, v_{x}$, and $v_{y}$, for $z_{0}=1000 \mathrm{~m}$ are shown in Fig. 10 . The derivatives are obtained using centered differencing

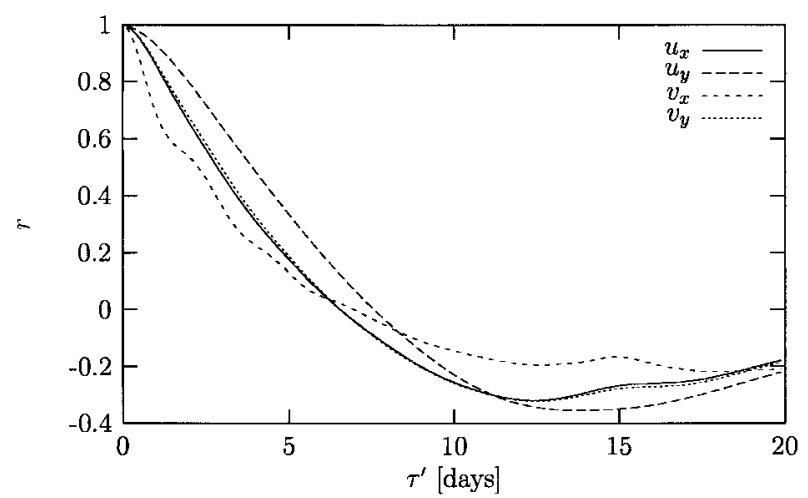

FIG. 10. Eulerian autocorrelations of the spatial derivatives for $z_{0}$ $=1000 \mathrm{~m}$. An average is taken over model grid points between $10^{\circ}$ and $60^{\circ} \mathrm{N}$.

of the velocities; ${ }^{6}$ the autocorrelation at a grid point of the model is calculated using (6); an average over grid points between $10^{\circ}$ and $60^{\circ} \mathrm{N}$ (an approximate cover of the surf zone) is taken. Autocorrelations of the velocities and of the principal strain rates are qualitatively similar.

From Fig. 10, there is little evidence of random strain in the Eulerian sense: the autocorrelations do not appear to decorrelate on the timescale of the integrations. One could, perhaps, argue that the zero crossing point corresponds to the correlation time, but this does not appear to be justified since the autocorrelations do not relax to zero, even when the integration is extended to 100 days. Quantification is therefore difficult, though for comparative purposes (see section $4 \mathrm{~b}$ ) we have tried, following the suggestion of a referee, to fit the autocorrelations to a function of the form

$$
r\left(\tau^{\prime}\right)=a \exp \left(-\tau^{\prime} / \tau_{c}\right) \cos \left(\omega \tau^{\prime}\right)
$$

where $a$ is a constant, $\tau_{c}$ is the correlation time, and $\omega$ a frequency. The fit so obtained is not very convincing, largely because the assumption of exponential decay does not appear to be valid. We have not tried to formalize this procedure through a nonlinear least-squares fitting because we feel that it would be rather pointless: the error in the assumed form (7) is probably comparable to that in the fitting parameters.

It should be noted in passing that given the evolution of the critical layer depicted in Fig. 2, this conclusion is to be expected: the velocity field is spatially smooth and evolves rather slowly.

There is similar behavior for other values of $z_{0}$; for brevity, only the autocorrelation of $u_{y}$ is shown (Fig.

\footnotetext{
${ }^{6}$ The first 10 days of the time series are excluded from the analysis since this is the time required for the forcing to reach maximum amplitude. This has no qualitative effect on the results.
} 


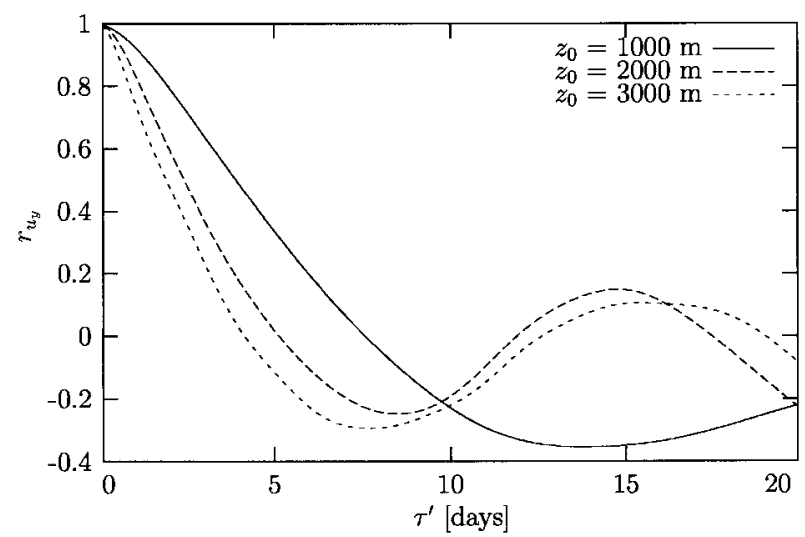

FIG. 11. Eulerian autocorrelation of $u_{y}$ for different values of the stationary forcing amplitude, $z_{0}$.

11). ${ }^{7}$ The autocorrelation for $z_{0}=3000 \mathrm{~m}$ is similar to the other cases, despite the splitting of the vortex (Fig. 4).

It is difficult to assign error bars to Figs. 10 and 11 because the standard error of $r_{u}\left(\tau^{\prime}\right)$ is of the same order of magnitude as $r_{u}\left(\tau^{\prime}\right)$ itself. The domain average implicit in the autocorrelation is taken over a large area, leading to a fairly broad distribution of "correlation times." (Ideally one would like to show the probability distribution of correlation times, but lacking an unambiguous method for determining them we adopt a domain average as a matter of course.) Nevertheless, the evolution seen above is not unrepresentative: the autocorrelations do not change substantially when the average is taken over a smaller area (not shown).

Time series for $z_{0}=2000 \mathrm{~m}$ (not shown) indicate that while the flow approaches a quasi-regular state, it does not appear to be quasiperiodic. This has been confirmed by computing power spectra of the time series: the spectra do not have characteristic peaks (cf. Rogerson et al. 1999 for an aperiodic case that does). In fact, the spectra appear to exhibit power-law scaling.

When stochastic forcing of sufficient amplitude is introduced, the velocity derivatives decorrelate rapidly and the Eulerian correlation time is unambiguously finite. Figure 12 shows $r_{u, v}$ for $\delta_{v}=0.01$ and $\delta_{v}=0.1$. For strong stochastic forcing $\left(\delta_{v}=0.1\right)$ Eulerian random strain unquestionably occurs: the autocorrelation fluctuates around zero for large $\tau^{\prime}$. For weak stochastic forcing $\left(\delta_{v}=0.01\right)$, the evolution is not much different from the deterministic case.

\section{b. Lagrangian correlation times}

Lagrangian autocorrelations, $r^{L}$, are computed for the shallow-water system using an initial particle distribu-

\footnotetext{
${ }^{7}$ Of the four derivatives, $u_{y}$ has the largest dimensional magnitude by far. For $z_{0}=3000 \mathrm{~m}$, differences between the derivatives do appear but the quasiperiodicity persists.
}

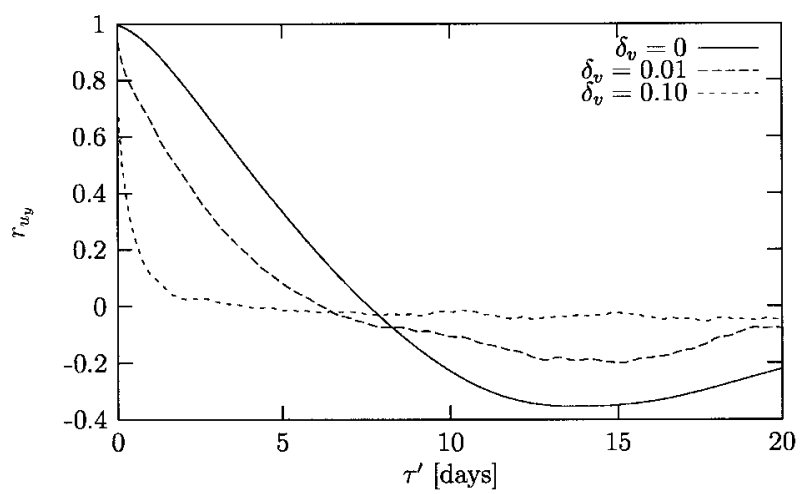

FIG. 12. Eulerian autocorrelation of $u_{\gamma}$ for continuous stochastic forcing in the velocity field; $z_{0}=1000 \mathrm{~m}$.

tion of 2000 points (doubling the number of particles has negligible effect on the results). As with the Eulerian autocorrelation the velocity derivatives are sampled every $3 \mathrm{~h}$, the time interval at which the velocity field is output. Velocity data from the entire time series (1200 $\mathrm{h}$ in the case of a 50-day integration) are used. ${ }^{8}$ To facilitate comparison a metric factor is applied in the computation of the $\lambda$ derivatives. The Lagrangian autocorrelations of the principal strain rates are similar to $r_{u}^{L}$.

Figure 13 shows Lagrangian autocorrelations for $z_{0}$ $=1000 \mathrm{~m}$. Here $u_{x}, v_{x}$, and $v_{y}$ clearly decorrelate along particle trajectories; $u_{y}$ decorrelates slowly, if at all. Although longer time series would be needed to establish that the velocity derivatives decorrelate completely, the contrast with the Eulerian autocorrelations, for which there is little sign of decorrelation, is clear enough. Coupled with the geometrical organization of the velocity field, we then conclude that chaotic advection does indeed occur.

The individual velocity derivatives exhibit different behavior because the velocity field is anisotropic: the integrations are initialized with a zonal jet, which persists even with strong stochastic forcing, explaining the slower decorrelation of $u_{y}$. This behavior differs from that of random-strain theory, where it is assumed that the elements of the strain matrix-and thus the individual velocity derivatives-decorrelate at a constant rate. Similar behavior arises in idealized chaotic systems (Ngan 1997; Dresselhaus and Tabor 1989) where it is often the case that some of the derivatives are time dependent, while others are time independent.

Despite the various pitfalls enumerated earlier, quantification of the correlation time is of some interest. Applying (7), one gets Eulerian correlation times $\tau_{E} \sim$ 10 days and Lagrangian correlation times $\tau_{L} \sim 2$ days.

\footnotetext{
${ }^{8}$ If the first 10 days of data were to be excluded, as with the Eulerian autocorrelations, then it is expected that decorrelation would be only more rapid.
} 


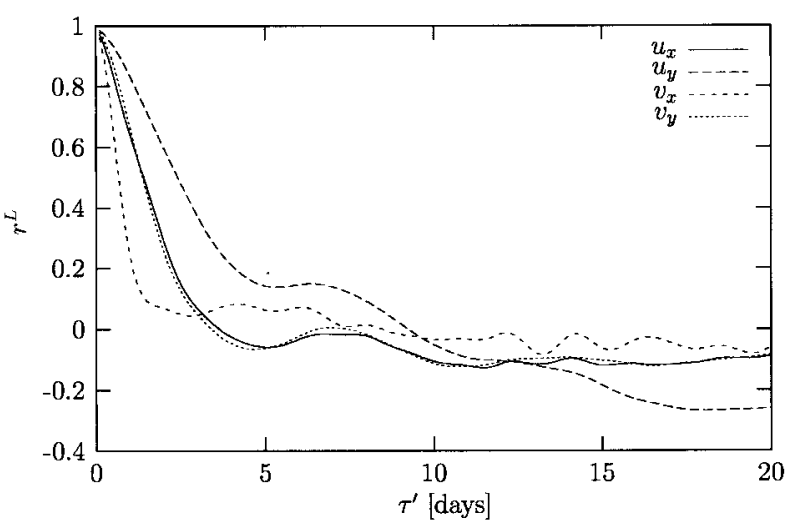

FIG. 13. Lagrangian autocorrelations for $u_{x}, u_{y}, v_{x}$, and $v_{y} ; z_{0}=$ $1000 \mathrm{~m}$

These numbers are very rough-each of the velocity derivatives decorrelates at a different rate, for example-but they do suggest that the ratio of $\tau_{E}$ to $\tau_{L}$ is of the order of 5. It would be hazardous to place too much faith in this number, but it is interesting that it is in agreement with the tracer scale factor of Methven and Hoskins (1999), which measures the ratio of scales accurately resolved by a contour advection simulation. Methven and Hoskins (1999) find that the tracer scale factor is $5.5 \pm 0.5$ in the Northern Hemisphere wintertime lower stratosphere.

There are similar results for other values of $z_{0}$ (Fig. 14). It would appear that the Lagrangian correlation time is determined primarily by the large-scale flow (the zonal-mean zonal velocity changes but slightly from $z_{0}=$ 1000 to $3000 \mathrm{~m}$ ); there is a similar phenomenon in the context of idealized Hamiltonian models, stochastic and deterministic (Ngan 1997). On the other hand, the Lagrangian autocorrelations do not exhibit long tails, which are a generic feature of Hamiltonian models, at least in the absence of noise (see, e.g., Badii et al. 1988). This is probably because there is greater time dependence in the spatial structure of the velocity field; consequently there is less long-range spatial order and weaker temporal correlation.

When stochastic forcing is introduced decorrelation is more rapid, though the decorrelation of $u_{y}$ still lags that of the other derivatives (Fig. 15). Although, strictly speaking, Lagrangian random strain still occurs, there is little sense in speaking of Lagrangian random strain (or chaotic advection) when both $\tau_{E}$ and $\tau_{L}$ are short: the flow is essentially quasi-turbulent.

\section{Calculations using isentropic winds from the Canadian Middle Atmosphere Model}

The robustness of the shallow-water results is now considered by performing a similar analysis using isentropic winds from the Canadian Middle Atmosphere Model (CMAM). CMAM is a general circulation model whose domain extends from the surface of the earth to (a)

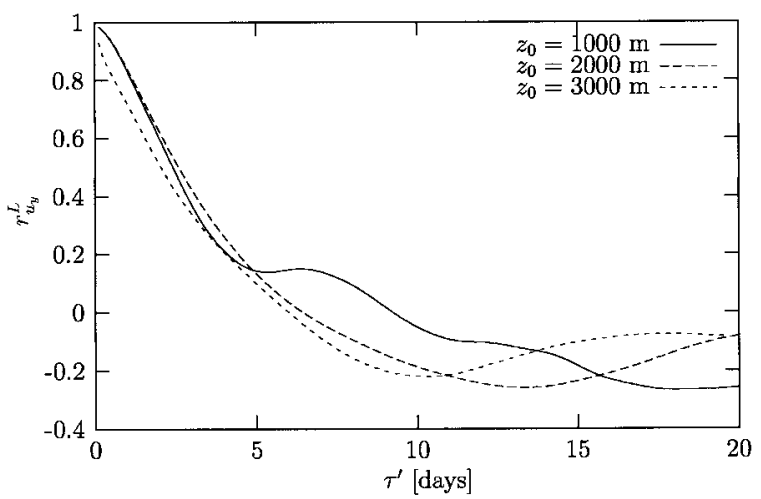

(b)

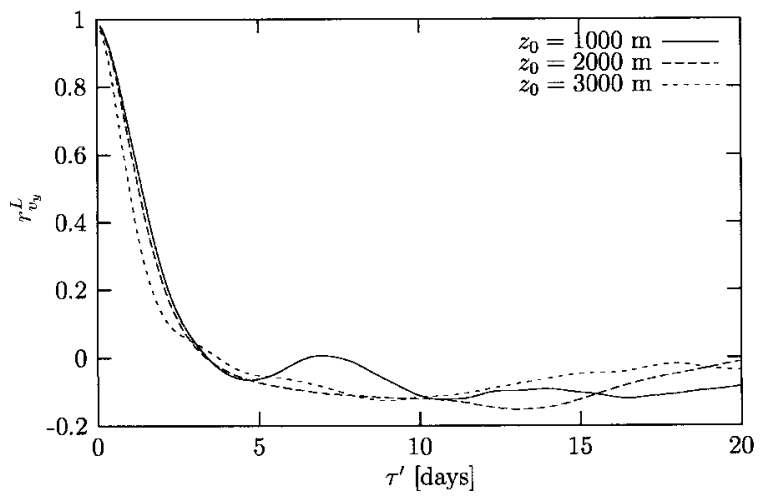

FIG. 14. Lagrangian autocorrelations for three different values of the stationary forcing amplitude, $z_{0}$. (a) $r_{u_{y}}^{L}$; (b) $r_{v_{y}}^{L}$.

about $95 \mathrm{~km}$; a detailed description may be found in Beagley et al. (1997). While CMAM is just a model, its complexity is such that results obtained from it should be broadly representative of the real stratosphere. (At the very minimum the stratospheric wave forcing should be more realistic than in the shallow-water model, large-scale and small-scale waves being present.) In principle, analyzed winds should be preferable to output from a numerical model, but as a balance constraint is inevitably imposed, it is not clear that this is so; gravity

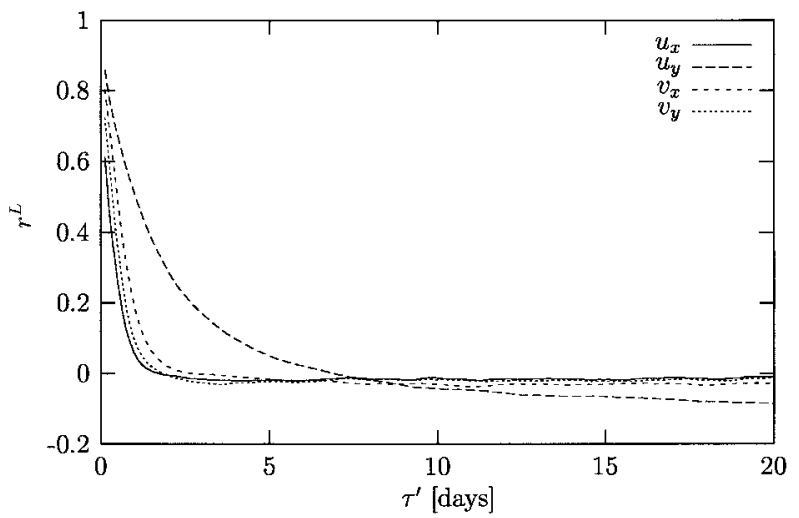

FIG. 15. Lagrangian autocorrelations for strong stochastic forcing of the velocity field $\left(\delta_{v}=0.05, z_{0}=250 \mathrm{~m}\right)$. 
waves, for example, must be parameterized (Pierce et al. 1994).

The winds are taken from 1 July to 30 July (i.e., Antarctic winter) of a long simulation using T32 horizontal resolution and 50 vertical levels between the ground and $0.001 \mathrm{hPa}$. Horizontal winds are output at 3 -h intervals onto a $65 \times 33$ grid with equally spaced grid points.

\section{a. Tracer fields}

Tracer fields are constructed using approximately 130000 points equally spaced in latitude and longitude from $5^{\circ}$ to $45^{\circ} \mathrm{S}$ at $10^{\circ}$ intervals and from $0.02^{\circ}$ to $359.98^{\circ} \mathrm{E}$ at $0.0125^{\circ}$ intervals. The integration procedure is identical to that used in section 3 .

Figure 16 shows tracer fields on three different isentropic levels at the end of a 30-day integration. The lowest level, $450 \mathrm{~K}$, is located at roughly $17 \mathrm{~km}$ and is often taken as being representative of the lower stratosphere; the highest level, $1000 \mathrm{~K}$, is located in the middle-stratosphere, at roughly $35 \mathrm{~km}$. In all cases cat's eyes in the surf zone may be seen, as in the shallowwater system. An interesting feature of this figure is that the wavenumber of the cat's eye structure decreases noticeably from 450 to $600 \mathrm{~K}$, though it remains approximately constant from 600 to $1000 \mathrm{~K}$. This is presumably a consequence of the Charney-Drazin theorem (e.g., Andrews et al. 1987), according to which, in an atmosphere with zonal velocity increasing monotonically with height, there is preferential filtering of smallscale waves (see also Scinocca and Haynes 1998). The strengthening of the basic flow may also play a role; recall that the morphology of the cat's eye structure in the shallow-water system is strongly affected by basicstate shear (Fig. 5). For the three isentropic surfaces considered here characteristic initial zonal velocities are 50,80 , and $130 \mathrm{~m} \mathrm{~s}^{-1}$.

A few other comments are also in order. First, the picture at 10 and 20 days is qualitatively similar-cat's eyes are still present-though of course there is less small-scale structure. This is in contrast to the shallowwater system: the time development of a Rossby wave critical layer may be seen in Fig. 2 because the shallowwater model is initialized from a rest state at time $t=$ 0 ; this is not the case in CMAM. Second, a larger number of particles is required in the CMAM integrations because the unbalanced component of the motion is stronger and because potential vorticity is not conserved on the timescale of the integrations. This has the effect of smearing out particle trajectories, in a manner similar to, though much less pronounced than, Fig. 6. Nevertheless, the spatial organization of the large-scale flow in CMAM is undeniable.

\section{b. Correlation times}

Eulerian and Lagrangian autocorrelations are now computed following the procedure of section 4 . The only difference is that for the Eulerian autocorrelations the entire time series is now used (i.e., 30 days or $720 \mathrm{~h}$ ). The Eulerian autocorrelations are computed from $5^{\circ}$ to $55^{\circ} \mathrm{S}$; the Lagrangian autocorrelations use approximately 1700 points initially distributed over the same domain.

Figure 17 shows Eulerian and Lagrangian autocorrelations for the isentropic levels shown in Fig. 16. ${ }^{9}$ Once more there is strong evidence for Lagrangian random strain (and chaotic advection): as with the shallowwater system (cf. Figs. 10 and 13), the Lagrangian correlation time is distinctly shorter than the Eulerian correlation time. Quantifying this once more with (7), one gets that $\tau_{E} \sim 4$ days and $\tau_{L} \sim 1$ day. Although both $\tau_{E}$ and $\tau_{L}$ are shorter than is the case for the shallowwater model, their ratio is approximately the same.

Another interesting point is that $\tau_{L}$ is of the same order of magnitude as the upper bound on contour advection sampling times $(\sim 12 \mathrm{~h}$ in the lower stratosphere). ${ }^{10}$ This timescale has been explained as the characteristic time associated with the advection of PV filaments in the surf zone (Waugh and Plumb 1994), which is a rough constraint on the accuracy of the simulated filaments' position. It is reasonable that this timescale be comparable to the Lagrangian correlation time.

The behavior for the three isentropic levels is similar and $\tau_{E}$ and $\tau_{L}$ are largely unchanged. The main difference is that fast oscillations are more prominent at higher altitudes. This behavior has been corroborated by the associated time series.

\section{Conclusions}

In this work we have tried to clarify what is meant by the commonly invoked, though rarely defined, phrase "chaotic advection in the stratosphere." This has been done by studying (i) the large-scale structure of the velocity field, specifically as manifested in tracer and potential vorticity fields, and (ii) the contrast between temporal Eulerian and Lagrangian velocity autocorrelations. Essentially we have tried to look for analogs of the defining features of chaotic advection in kinematic models-namely, suitable geometric organization of the large-scale flow and temporal "quasi-regularity"which apply even when the flow is not periodic or quasiperiodic in time.

The most immediate (and obvious) conclusion of this work is that chaotic advection does, in all likelihood, occur in the wintertime stratosphere. By itself, this conclusion is not very surprising. The existence of suitable

\footnotetext{
${ }^{9}$ For brevity we show autocorrelations of $u_{y}$ only. The behavior of the other derivatives is analogous to that of the shallow-water system (see Figs. 10 and 13), i.e., they decorrelate more rapidly than $u_{y}$. However, the contrast between the Eulerian autocorrelations is noticeably smaller at 600 and $1000 \mathrm{~K}$.

${ }^{10}$ We thank Lynn Sparling for bringing this point to our attention.
} 
(a)

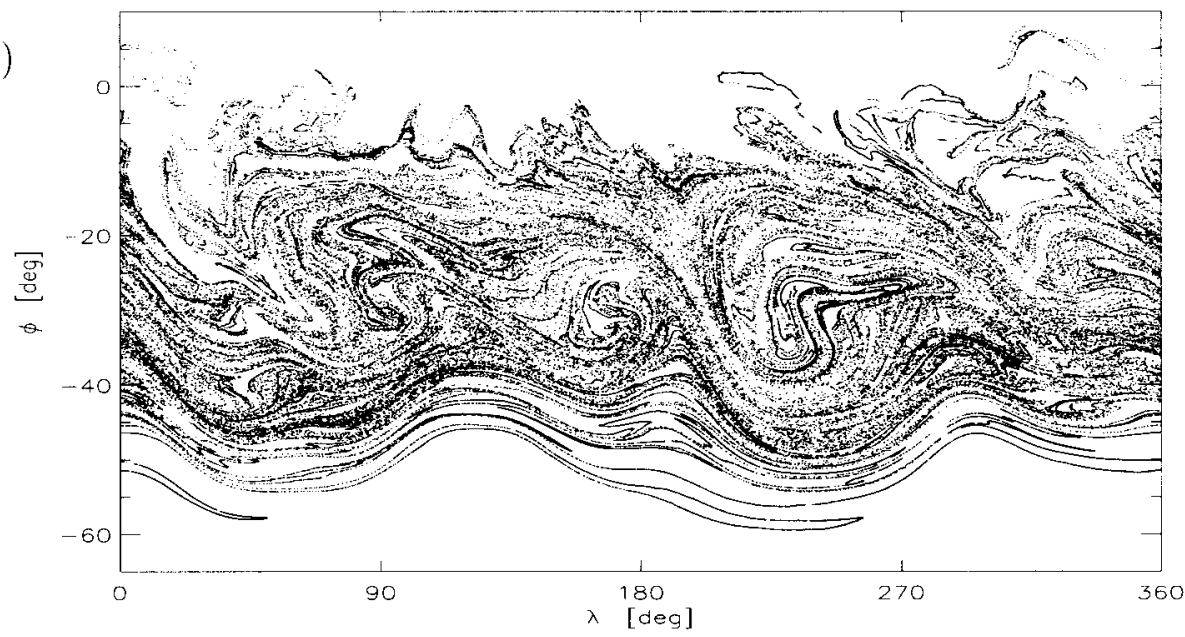

(b)

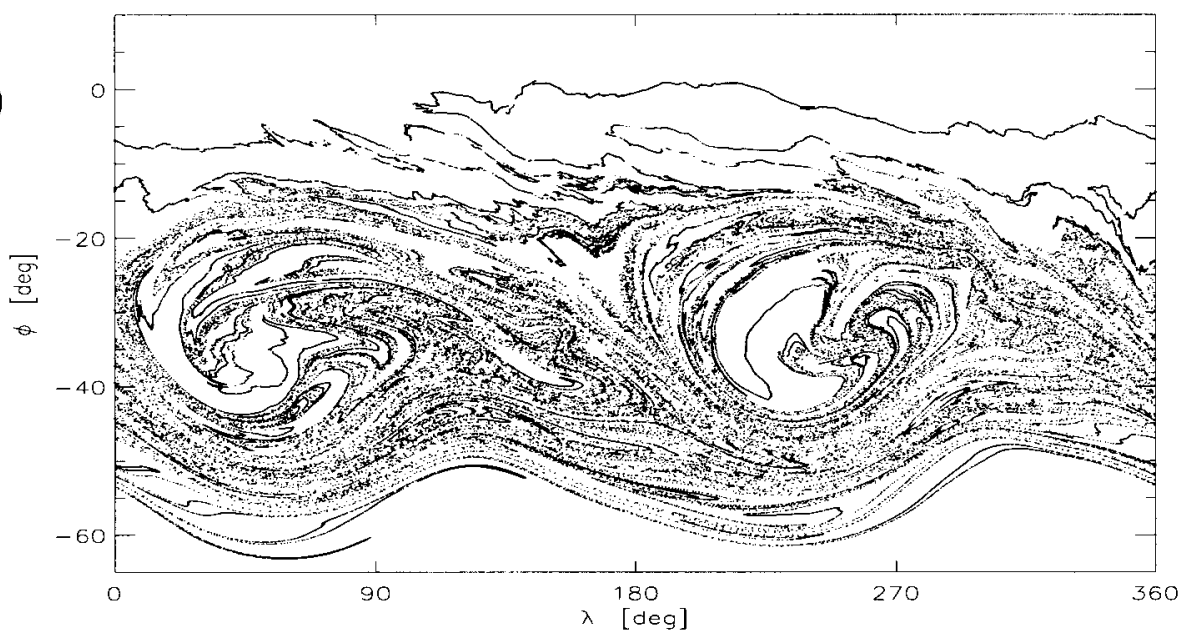

(c)

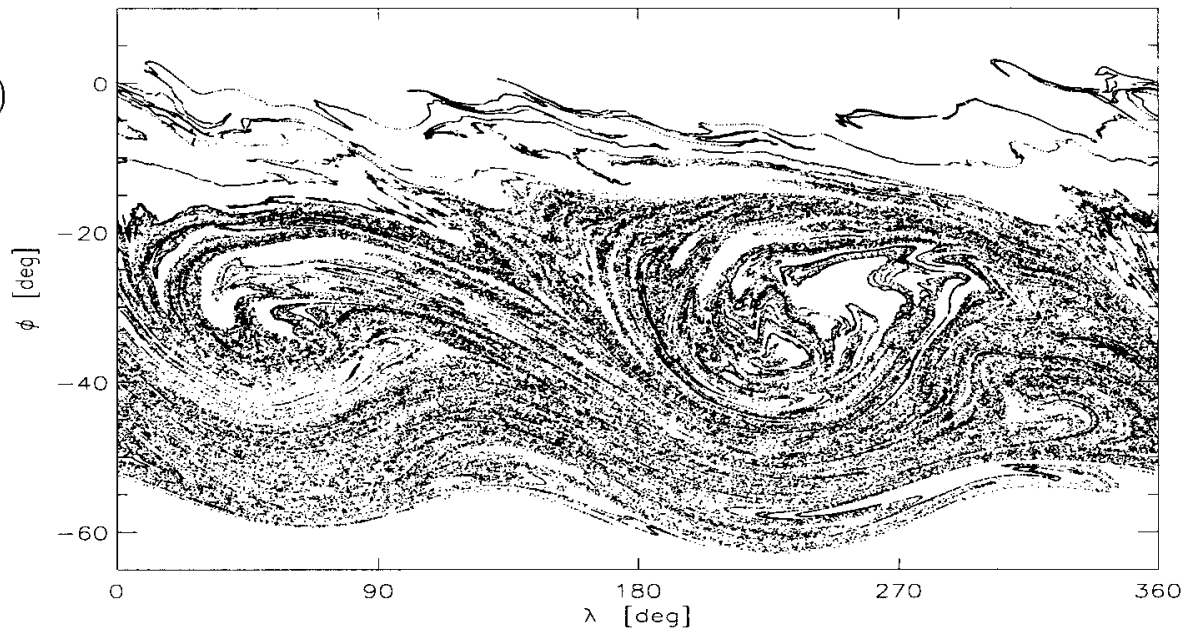

FIG. 16. Tracer fields after a 30-day integration using isentropic winds from CMAM. (a) $\theta=450 \mathrm{~K}$; (b) $\theta=600 \mathrm{~K}$; (c) $\theta=1000 \mathrm{~K}$. 

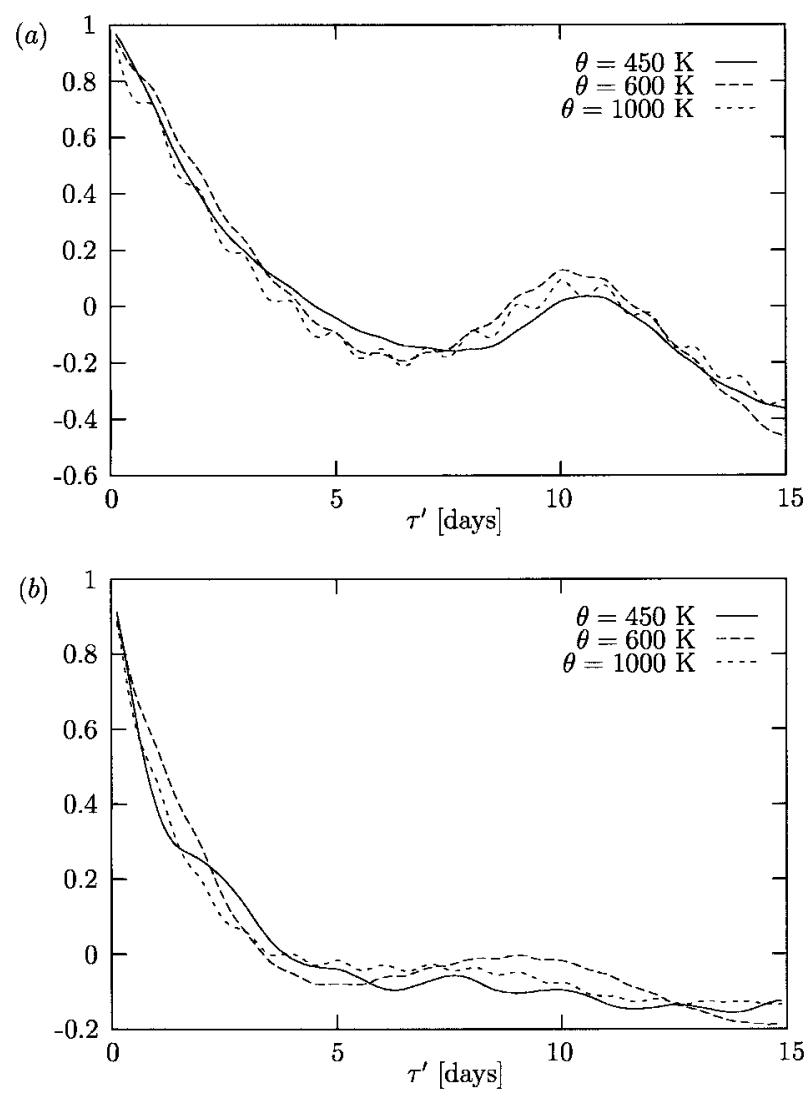

FIG. 17. Autocorrelations of the velocity derivatives on the $\theta=$ 450,600 , and $1000 \mathrm{~K}$ isentropic surfaces. (a) $r_{u_{y}}$; (b) $r_{u_{y^{*}}}^{L}$

large-scale structure in the velocity field is implied by the work of Waugh and Plumb (1994) and Norton (1994), among others; calculations by several authors (e.g., Bowman 1993a; Waugh and Plumb 1994; Schoeberl and Newman 1995) have shown that particle trajectories in the surf zone are chaotic. Yet while the occurrence of chaotic advection in the stratosphere is not especially surprising, it is useful, we believe, to have posed such a question in the first place. Apart from resolving this question, in trying to understand how chaotic advection is of relevance to stratospheric mixing and transport we learn a number of things about them both.

First, we have shown how the behavior of the barotropic Rossby wave critical layer, which is precisely defined for small forcing amplitude only, naturally extends to finite amplitude and nonbarotropic flow. The emergence of cat's eye structures in both the shallowwater system and the middle-atmosphere general circulation model (CMAM) has been emphasized. We find that such structures exist when stochasticity is sufficiently weak, as is the case in the stratosphere. This is the regime where chaotic advection is applicable.

Second, the notion of an organizing structure for mixing and transport may prove useful in accounting for the existence of chaotic advection in intrinsically dy- namical, vorticity-conserving fluids. As it is well known that kinematic models of chaotic advection in geophysical fluids suffer from a dynamical consistency problem (see Ngan and Shepherd 1997 and references therein), there has been some debate over their applicability. Briefly, this dynamical consistency problem arises from the fact that these models, which are essentially Hamiltonian dynamical systems in the guise of two-dimensional incompressible flow, may not, in general, materially conserve potential vorticity. The notion of an organizing structure establishes an explicit connection between this physical constraint on chaotic advection in a vorticity-conserving fluid and the mathematical constraint on chaotic advection in a temporally aperiodic system (i.e., the existence of a well-defined heteroclinic structure): the finite-amplitude Rossby wave critical layer is the direct analog of the aperiodic heteroclinic structure.

Of course it would be more satisfying if the manifolds were calculated directly; however, this appears to be a difficult calculation. Malhotra and Wiggins (1998) computed manifolds for an aperiodic flow but one that is kinematically specified, so that the position of the hyperbolic points is known exactly, greatly simplifying the calculation. Miller et al. (1997) computed manifolds for a dynamically consistent flow, but their analysis exploits the fact that the flow is almost quasiperiodic, allowing them to work in a comoving frame and obtain well-defined manifolds. Rogerson et al. (1999) were able to compute manifolds for a fully aperiodic flow, but the strong hyperbolicity of the flow was critical to the success of their calculation. As has been shown by Haller and Poje (1998) and Poje and Haller (1999), manifolds can be computed using finite time series if the flow satisfies certain technical requirements; nevertheless, the question remains as to how, in general, one locates the hyperbolic points in the first place. If the flow is aperiodic and most trajectories are, from a numerical standpoint, at least partially hyperbolic, then a distinguished hyperbolic trajectory may not be easy to identify.

Third, we have tried to clarify the connections between chaotic advection, stratospheric mixing, and random-strain theory. Both random-strain theory and chaotic advection have been invoked as explanations of stratospheric mixing, and while there are many similarities between them, they are not equivalent. The fundamental difference is that random strain is a more general phenomenon than chaotic advection, for it is purely statistical in nature; the concepts of Eulerian and Lagrangian random strain were introduced in order to help make this distinction clear. Computations of Eulerian and Lagrangian autocorrelations for the shallow-water system and CMAM indicate that Lagrangian random strain occurs: velocity derivatives decorrelate more rapidly along particle trajectories than at fixed locations. More interestingly, perhaps, the ratio of Eulerian to Lagrangian correlation times for both models is about 5; 
this is close to the tracer-scale factor of Methven and Hoskins (1999), which describes the range of scales accurately simulated by contour advection. This ratio is, moreover, approximately independent of height in CMAM.

None of which is to say that the shallow-water system or the middle-atmosphere model obey all the formal requirements of random-strain theory. Most glaringly, the velocity derivatives do not, at least on the timescales we have considered, decorrelate at precisely the same rate. In the second part of this study we provide examples of how chaotic advection in the stratosphere does indeed yield statistical behavior in accord with that of random-strain theory. The basic idea is that randomstrain theory extends beyond its formal range of applicability because the underlying physical mechanism is robust: generation of small-scale tracer structure by a large-scale strain field. Indeed, many chaotic systems fall into this category.

Acknowledgments. The authors thank S. Gravel and M. Roch of Recherche en Prévision Numérique (Environment Canada) for providing the shallow-water model, J. N. Koshyk for the CMAM data, and T. D. A. Fairlie, J. Methven, L. M. Polvani, and L. C. Sparling for helpful discussion and comments. We also thank R. A. Plumb for many constructive criticisms, and an anonymous referee for suggesting that the correlation times be computed explicitly. This work was supported by the Natural Sciences and Engineering Research Council and the Atmospheric Environment Service of Canada.

\section{REFERENCES}

Andrews, D. G., J. R. Holton, and C. B. Leovy, 1987: Middle Atmosphere Dynamics. Academic Press, 489 pp.

Antonsen, T. M., Z. Fan, E. Ott, and E. Garcia-Lopez, 1996: The role of chaotic orbits in the determination of power spectra of passive scalars. Phys. Fluids, 8, 3094-3104.

Badii, R., K. Heinzelmann, P. F. Meier, and A. Politi, 1988: Correlation functions and generalized Lyapunov exponents. Phys. Rev. A, 37, 1323-1328.

Batchelor, G. K., 1952: The effect of homogeneous turbulence on material lines and surfaces. Proc. Roy. Soc. London A, 213, 349366.

_ 1959 : Small-scale variation of convected quantities like temperature in turbulent fluid. Part 1, General discussion and the case of small conductivity. J. Fluid Mech., 5, 113-133.

Beagley, S. R., J. de Grandpré, J. N. Koshyk, N. A. McFarlane, and T. G. Shepherd, 1997: Radiative-dynamical climatology of the first-generation Canadian Middle Atmosphere Model. Atmos.Ocean, 35, 293-331.

Beigie, D., A. Leonard, and S. Wiggins, 1994: Invariant manifold templates for chaotic advection. Chaos Applied to Fluid Mixing, H. Aref and M. S. El Naschie, Eds., Pergamon Press, 5-124.

Bowman, K. P., 1993a: Large-scale isentropic mixing properties of the Antarctic polar vortex from analyzed winds. J. Geophys. Res., 98, 23 013-23 027.

_ 1993b: Barotropic simulation of large-scale mixing in the Antarctic polar vortex. J. Atmos. Sci., 50, 2902-2914.

_, 1996 : Rossby wave phase speeds and mixing barriers in the stratosphere. Part I: Observations. J. Atmos. Sci., 53, 905-916.

Chertkov, M., G. Falkovich, I. Kolokolov, and I. Lebedev, 1995:
Statistics of a passive scalar advected by a large-scale two-dimensional velocity field: Analytic solution. Phys. Rev. E, 51, $5609-5627$.

Côté, J., M. Roch, A. Staniforth, and L. Fillion, 1993: A variableresolution semi-Lagrangian finite-element global model of the shallow-water equations. Mon. Wea. Rev., 121, 231-243.

Crisanti, A., and A. Vulpiani, 1993: On the effects of noise and drift on diffusion in fluids. J. Stat. Phys., 70, 197-211.

Dresselhaus, E., and M. Tabor, 1989: The persistence of strain in dynamical systems. J. Phys. A, 22, 971-984.

Dritschel, D. G., P. H. Haynes, M. N. Juckes, and T. G. Shepherd, 1991: The stability of a two-dimensional vorticity filament under uniform strain. J. Fluid Mech., 230, 647-665.

Haller, G., and A. C. Poje, 1998: Finite time transport in aperiodic flows. Physica D, 119, 352-380.

Haynes, P. H., 1987: On the instability of sheared disturbances. $J$. Fluid Mech., 175, 463-478.

, 1989: The effect of barotropic instability on the nonlinear evolution of a Rossby-wave critical layer. J. Fluid Mech., 207, 231266.

_ , and M. E. McIntyre, 1987: On the representation of Rossbywave critical layers and wave breaking in zonally truncated models. J. Atmos. Sci., 44, 2359-2382.

— , and J. Anglade, 1997: The vertical-scale cascade in atmospheric tracers due to large-scale differential advection. J. Atmos. Sci., 54, 1121-1136.

Held, I. M., and P. J. Phillips, 1987: Linear and nonlinear barotropic decay on the sphere. J. Atmos. Sci., 44, 200-207.

Ishioka, K., and S. Yoden, 1995: Non-linear aspects of a barotropically unstable polar vortex in a forced-dissipative system: Flow regimes and tracer transport. J. Meteor. Soc. Japan, 73, 201212 .

Jana, S. C., G. Metcalfe, and J. M. Ottino, 1994: Experimental and computational study of mixing in complex Stokes flow: The vortex mixing flow and multicellular cavity flows. J. Fluid Mech., 269, 199-246.

Juckes, M. N., 1989: A shallow water model of the winter stratosphere. J. Atmos. Sci., 46, 2934-2955.

— of breaking planetary waves in the stratosphere. Nature, 328, 590-596.

Killworth, P. D., and M. E. McIntyre, 1985: Do Rossby-wave critical layers absorb, reflect or over-reflect? J. Fluid Mech., 161, 449492.

Kraichnan, R. H., 1974: Convection of a passive scalar by a quasiuniform random straining field. J. Fluid Mech., 64, 737-762.

Malhotra, N., and S. Wiggins, 1998: Geometric structures, lobe dynamics, and Lagrangian transport in flows with aperiodic timedependence, with applications to Rossby wave flow. J. Nonlinear Sci., 8, 401-456.

McCalpin, J. D., 1988: A quantitative analysis of the dissipation inherent in semi-Lagrangian advection. Mon. Wea. Rev., 116, $2330-2336$.

Methven, J., and B. Hoskins, 1999: The advection of high-resolution tracers by low-resolution winds. J. Atmos. Sci., 56, 3262-3285.

Miller, P. D., C. K. R. T. Jones, A. M. Rogerson, and L. J. Pratt, 1997: Quantifying transport in numerically generated velocity fields. Physica D, 110, 105-122.

Ngan, K., 1997: Chaotic advection in the stratosphere. Ph.D. thesis, University of Toronto, $179 \mathrm{pp}$.

- , and T. G. Shepherd, 1997: Chaotic mixing and transport in Rossby-wave critical layers. J. Fluid Mech., 334, 315-351.

— stratosphere. Part II: Statistical diagnostics. J. Atmos. Sci., 56, 4153-4166.

Norton, W. A., 1994: Breaking Rossby waves in a model stratosphere diagnosed by a vortex-following coordinate system and a technique for advecting material contours. J. Atmos. Sci., 51, 654673. 
Ottino, J. M., 1989: The Kinematics of Mixing: Stretching, Chaos and Transport. Cambridge University Press, 364 pp.

Pierce, R. B., and T. D. A. Fairlie, 1993: Chaotic advection in the stratosphere: Implications for the dispersal of chemically perturbed air from the polar vortex. J. Geophys. Res., 98, $18589-$ 18595.

- - W. L. Grose, R. Swinbank, and A. O'Neill, 1994: Mixing processes within the polar night jet. J. Atmos. Sci., 51, 29572972.

Pierrehumbert, R. T., 1994: Tracer microstructure in the large-eddy dominated regime. Chaos Applied to Fluid Mixing, H. Aref and M. S. El Naschie, Eds., Pergamon Press, 347-366.

Poje, A. C., and G. Haller, 1999: Geometry of cross-stream mixing in a double-gyre ocean model. J. Phys. Oceanogr., 29, 16491665.

Polvani, L. M., D. W. Waugh, and R. A. Plumb, 1995: On the subtropical edge of the stratospheric surf zone. J. Atmos. Sci., 52, 1288-1309.

Randel, W. J., and I. M. Held, 1991: Phase speed spectra of transient eddy fluxes and critical layer absorption. J. Atmos. Sci., 48, 688697.

Rogerson, A. M., P. D. Miller, L. J. Pratt, and C. K. R. T. Jones, 1999: Lagrangian motion and fluid exchange in a barotropic meandering jet. J. Phys. Oceanogr., 29, 2635-2655.

Salby, M. L., 1992: The influence of planetary-wave transience on horizontal air motions in the stratosphere. J. Atmos. Sci., 49, 405-421.

- , and R. R. Garcia, 1987: Vacillations induced by interference of stationary and traveling planetary waves. J. Atmos. Sci., 44, 2679-2711

— - D. O'Sullivan, and J. Tribbia, 1990a: Global transport calculations with an equivalent barotropic system. J. Atmos. Sci., 47, 188-214.

- D. O'Sullivan, R. R. Garcia, and P. Callaghan, 1990b: Air motions accompanying the development of a planetary wave critical layer. J. Atmos. Sci., 47, 1179-1204.

Schoeberl, M. R., and P. A. Newman, 1995: A multiple-level trajectory analysis of vortex filaments. J. Geophys. Res., 100, 25 80125815.

Scinocca, J. F., and P. H. Haynes, 1998: Dynamical forcing of planetary waves by tropospheric baroclinic eddies. J. Atmos. Sci., 55, 2361-2392.

Sutton, R. T., H. Maclean, R. Swinbank, A. O'Neill, and F. W. Taylor, 1994: High-resolution stratospheric tracer fields estimated from satellite observations using Lagrangian trajectory calculations. J. Atmos. Sci., 51, 2995-3005.

Swanson, K. L., and R. T. Pierrehumbert, 1997: Lower-tropospheric heat transport in the Pacific storm track. J. Atmos. Sci., 54, 15331543.

Tuck, A. F, and Coauthors, 1997: The Brewer-Dobson circulation in the light of high-altitude in-situ aircraft observations. Quart. J. Roy. Meteor. Soc., 123, 1-69.

Waugh, D. W., and D. G. Dritschel, 1991: The stability of filamentary vorticity in two-dimensional geophysical vortex-dynamics models. J. Fluid Mech., 231, 575-598.

_ , and R. A. Plumb, 1994: Contour advection with surgery: A technique for investigating fine-scale structure in tracer transport. J. Atmos. Sci., 51, 530-540.

Wiggins, S., 1990: Introduction to Applied Nonlinear Dynamical Systems and Chaos. Springer, 672 pp.

- 1992: Chaotic Transport in Dynamical Systems. Springer, 301 pp. 